\title{
MATHEMATICAL ANALYSIS OF AN AGE STRUCTURED EPIDEMIC MODEL WITH A QUARANTINE CLASS
}

\author{
ZAKYA SARI $^{1}$, Tarik Mohammed Touaoula ${ }^{1}{ }^{1}$ \\ AND BedREDDine AinsebA ${ }^{2, *}{ }^{\circ}$
}

\begin{abstract}
In this paper, an age structured epidemic Susceptible-Infected-Quarantined-RecoveredInfected (SIQRI) model is proposed, where we will focus on the role of individuals that leave the $R$-class before being completely recovered and thus will participate again to the disease transmission. We investigate the asymptotic behavior of solutions by studying the stability of both trivial and positive equilibria. In order to see the impact of the different model parameters like the relapse rate on the qualitative behavior of our system, we firstly, give an explicit expression of the basic reproduction number $R_{0}$, which is a combination of the classical basic reproduction number for the SIQR model and some other model parameters, corresponding to the individuals infected by the relapsed ones. It will be shown that, if $R_{0} \leq 1$, the disease free equilibrium is globally asymptotically stable and becomes unstable for $R_{0}>1$. Secondly, while $R_{0}>1$, a suitable Lyapunov functional is constructed to prove that the unique endemic equilibrium is globally asymptotically stable on some subset $\Omega_{0}$.
\end{abstract}

Mathematics Subject Classification. 58F15, 58F17, 53C35.

Received March 16, 2021. Accepted October 1, 2021.

\section{INTRODUCTION}

Understanding epidemic dynamics remains a major health problem nowadays. Mathematical modeling of epidemics has been increasingly classified as a powerful tool to understand various interactions between the transmission dynamics and some other parameters controlling it, like demographical parameters, size of the population, control actions, etc.

This paper will focus on an epidemic model of Susceptible-Infected-Quarantined-Recovered-Infected (SIQRI) type where we will look for the role of individuals that leave the $R$-class before being completely recovered and thus will participate again to the transmission of the disease. We will also assume that all individuals completely respect the quarantine, which is idealistic but can hold true; for example in a disciplined population, or if the quarantine is not very restrictive. But we assume that they can relaps once they are recovered.

One of the leading works on epidemic mathematical models was introduced by Bernouilli in 1760's in [6, 7]. Very later, Kermack and McKendrik proposed the first susceptible-infected- recovered (SIR) model [20]. Since

Keywords and phrases: Age structure, SIQRI model, relapse rate, global stability, persistence, basic reproductive number,

Lyapunov functional.

${ }^{1}$ Laboratoire d'Analyse Non linéaire et Mathématiques Appliquées, Département de Mathématiques, Université Aboubekr Belkaïd, Tlemcen 13000, Algérie.

2 Institut de Mathématiques de Bordeaux, IMB UMR CNRS 5251, Université de Bordeaux, 33000, Bordeaux, France.

* Corresponding author: bedreddine.ainseba@u-bordeaux.fr 
then, many epidemic models like SIQR model among others have been developed in ODEs equations, see for instance $[9,16]$ and references therein.

The chronological age as a variable that can play an important role in the epidemic has been introduced in $[18,32]$ and the references therein. Further, the infection age (the elapsed time since the start of the infection) structure, was considered in various works, [4, 8, 9, 12, 15, 23, 24, 29]. See also SIR model [11], SIRS model [13], SIQ model [17], SEIRS model [33]. These models consider some parameters like the incidence rate which depends on the nature of the transmission of the infection. For various mathematical forms of an incidence rate, see for instance $[2,5,11,17]$, and references therein.

Epidemiologically, among all control actions that could change the transmission, the screening strategy is known as the best intervention that could identify very early the infected individuals $[1,2]$ for treatment or isolation. However, the population functional response must be taken into account, in particular non linear ones, which makes the model not easy to handle. For the non linear epidemic model see $[2,5]$ and the references therein.

Once identified, the quarantining measure is needed as a first health action for containing the disease, [17]. Because of the efficiency of the treatments and the long stay period in the quarantine class some individuals stop their treatments or modify their habits [25]. Consequently they are relapsed and replaced into the infected class again. For a mathematical model with relapse see $[11,17]$. The last case can occur for the tuberculosis disease which is known as a bacterial contagious disease $[10,25]$ for a preventive therapy. However, as it is detailed in [1] this disease is characterized by its long time latency period (the period between starting and communicating the disease). For recent contributions of tuberculosis disease dynamics, see [3, 31].

In [24], the authors considered an age structured SI epidemic model with an infection age in the infected class and uses a Lyapunov function to obtain the global stability of the unique endemic equilibrium. In [21] an age structured SIR model in each healthy subclass is considered. The author reduces the model into a four dimensional system of ODEs and performs a fourth order characteristic equation to prove the local stability of the endemic equilibrium. In [33] an age structured SEIRS model is reduced to a non linear delayed system to obtain the local stability of both trivial and positive equilibrium. In [11], a SIR model including the infection age in the infected class is studied, while in [17] a SIQ one includes it in the infected and in the quarantine classes. Both of them include a non linear incidence function and relapse, for each one the global stability is proved in a Lyapunov sense. In [2], the optimal control is investigated for an SIR type model with an infection age structure uniquely in the infected class and a screening strategy depending only on the time. In most of all these works, the reproduction rate $R_{0}$ is computed and compared to the unity which determines the behavior of the systems. In this context, see [14]. The persistence results are then established if $R_{0}$ is greater than unity, see $[27]$.

In the present paper, we propose a non linear model of a SIQRI type with an infection age structure in both infected and quarantined classes, where the screening function depends on the age. Our model would be an extension to the one proposed in [2]. We believe that, such a model may represent among other disease the TB infection dynamics.

Our model takes into account what follows:

- A fraction of the susceptible individuals enters into the infected class once the disease is transmitted until they are screened.

- A subfraction of the infected individuals once detected, is quarantined for treating.

- Some of them are removed, while others are relapsed and then considered as newly infected i.e. the infected with the infection age zero.

We firstly compute the basic reproductive rate in order to clarify its dependency with respect to each parameter in a combined screening/population response/quarantining process, regulating the behaviour of our system. Secondly, the stability is investigated in the Lyapunov sense.

This work is organized as follows: Section 2 is devoted to the statement of the problem, Section 3 is concerned by the existence theorem of a compact attractor. In Section 4, the local and the global stability of a free disease 
equilibrium are shown. The persistence results and the global stability of the endemic equilibrium are proved in Section 5. To illustrate our results, numerical simulations are given in Section 6.

\section{THE MODEL}

We consider a host population divided in four sub-populations: the susceptible, the infected, the quarantined and the recovered classes. The densities of individuals of each class are denoted respectively by $S(t), i(t, a)$, $q(t, a)$ and $R(t)$, where $a \geq 0$ is the infection age (the duration time since infection) at each time $t \geq 0$. The model is given by the following system

$$
\left\{\begin{array}{l}
S^{\prime}(t)=A-\mu S(t)-S(t) J(t) \\
\frac{\partial i(t, a)}{\partial t}+\frac{\partial i(t, a)}{\partial a}=-\mu i(t, a)-v(a) \psi(N(t)) i(t, a), \\
\frac{\partial q(t, a)}{\partial t}+\frac{\partial q(t, a)}{\partial a}=v(a) \psi(N(t)) i(t, a)-(\mu+\phi(a)) q(t, a), \\
R^{\prime}(t)=\int_{0}^{\infty} \phi(a) q(t, a) \mathrm{d} a-(\mu+\delta) R(t), \\
i(t, 0)=S(t) J(t)+\delta R(t), \\
q(t, 0)=0, \\
J(t)=\int_{0}^{\infty} \beta(a) i(t, a) \mathrm{d} a
\end{array}\right.
$$

with $N(t)=S(t)+\int_{0}^{\infty} i(t, a) \mathrm{d} a+\int_{0}^{\infty} q(t, a) \mathrm{d} a+R(t)$. This system is completed by the following initial data

$$
\left\{\begin{array}{l}
S(0)=S_{0}, \quad R(0)=r_{0}, \\
i(0, a)=i_{0}(a), \quad i_{0} \in L^{1}\left(\mathbb{R}^{+}, \mathbb{R}^{+}\right), \\
q(0, a)=q_{0}(a), \quad q_{0} \in L^{1}\left(\mathbb{R}^{+}, \mathbb{R}^{+}\right) .
\end{array}\right.
$$

The parameters $A, \mu$, are respectively the flux entering to the susceptible class and the natural death rate of the host population. The parameters in (2.1) have the following description: $\delta$ : per capita relapse rate. $v(a)$ : screening strategy, i.e. the planned fraction of individuals to be screened per unit of age; $\psi(z)$ : populationdependent individual functional response to the screening offer, i.e. the fraction of individuals accepting the screening offer; $\phi(a)$ : age dependent removal term; $\beta(a)$ : age dependent transmission rate; $J(t)$ : force of infection per unit of time $t$.

Throughout this paper we assume that all data of (2.1) are positive and

(H0) $\beta \in C_{B U}\left(\mathbb{R}^{+} ; \mathbb{R}^{+}\right)$, where $C_{B U}\left(\mathbb{R}^{+} ; \mathbb{R}^{+}\right)$is the set of bounded uniformly continuous functions.

(H1) $\psi \in C\left(\mathbb{R}^{+} ; \mathbb{R}^{+}\right)$and $v \in \mathrm{E}^{\infty}\left((0, \infty) ; \mathbb{R}^{+}\right)$.

(H2) $\phi \in \mathrm{E}^{\infty}\left((0, \infty) ; \mathbb{R}^{+}\right) \cap \mathrm{E}^{1}\left((0, \infty) ; \mathbb{R}^{+}\right)$with $\int_{0}^{\infty} \phi(a) \mathrm{d} a=1$.

Note that the functional response $\Psi(N) N$ is the part of the population accepting screening and $\frac{i(t, a) \mathrm{d} a}{N(t)}$ is the probability that the screened individual is infective. The functional response $\Psi(z) z$ will be taken as a Holling type function in the numerical section so that $\Psi(z)$ will be a decreasing function and $\Psi(z) z$ a saturating increasing function (see for instance [2] for more details in the parameters description). 
We define the normed space $\left(X,\|.\|_{X}\right)$ as

$$
\begin{gathered}
X:=\mathbb{R} \times\left(L^{1}(0, \infty ; \mathbb{R})\right)^{2} \times \mathbb{R} \\
\|(S(t), i(t, .), q(t, .), R(t))\|_{X}=|S(t)|+\|i(t, .)\|_{1}+\|q(t, .)\|_{1}+|R(t)|,
\end{gathered}
$$

with $\|f\|_{1}=\int_{0}^{\infty}|f(x)| \mathrm{d} x$. The associated positive convex cone is

$$
X^{+}:=\mathbb{R}^{+} \times\left(L^{1}\left(0, \infty ; \mathbb{R}^{+}\right)\right)^{2} \times \mathbb{R}^{+} .
$$

Using a standard methods, (fixed point methods) it is possible to prove existence and uniqueness of a non-negative solution to system 2.1, see also [5].

Integrating along the characteristic lines, we find,

$$
i(t, a)= \begin{cases}i(t-a, 0) \pi_{1}(t-a, a), & \text { if } t>a, \\ i_{0}(a-t) \frac{\pi_{1}(t-a, a)}{\pi_{1}(t-a, a-t)}, & \text { if } t<a .\end{cases}
$$

Using (2.3), see also [18], we obtain,

$$
q(t, a)=\left\{\begin{array}{l}
i(t-a, 0) \int_{0}^{a} v(s) \psi(N(s+t-a)) \frac{\pi_{2}(a)}{\pi_{2}(s)} \pi_{1}(t-a, s) \mathrm{d} s, \text { if } t>a, \\
\frac{\pi_{2}(a)}{\pi_{2}(a-t)} q_{0}(a-t) \\
+i_{0}(a-t) \frac{\pi_{2}(a)}{\pi_{1}(t-a, a-t)} \int_{a-t}^{a} v(s) \psi(N(s+t-a)) \frac{\pi_{1}(t-a, s)}{\pi_{2}(s)} \mathrm{d} s, \text { if } t<a,
\end{array}\right.
$$

where

$$
\left\{\begin{array}{l}
\pi_{1}(t, a)=\exp \left(-\int_{0}^{a}(\mu+v(s) \psi(N(t+s))) \mathrm{d} s\right) \\
\pi_{2}(a):=\exp \left(-\int_{0}^{a}(\mu+\phi(s)) \mathrm{d} s\right)
\end{array}\right.
$$

are respectively the probabilities for an infected individual to stay in the infected class until age $a$ and for a quarantined individual to stay in the quarantine class until age $a$.

\section{Semiflow, compact attractor and total trajectories}

\subsection{Semiflow and compact attractor}

Setting $\phi_{0}=\left(S_{0}, i_{0}, q_{0}, r_{0}\right)$ and denoting by $\Phi\left(t, \phi_{0}\right)=(S(t), i(t,),. q(t,),. R(t))$ the semiflow passing through $\phi_{0}$.

Note that $(S(t), i(t,),. q(t,),. R(t))$ the solution of $(2.1)$ is time continuous and state continuous uniformly with respect to time.

For simplicity, throughout the paper we set $\bar{N}=\frac{A}{\mu}$. 
Theorem 3.1. For $\phi_{0}$ in $X^{+}$, there exists a unique positive solution of (2.1) in $X^{+}$. Moreover, we have

$$
N(t) \leq \max (N(0), \bar{N})
$$

and

$$
\lim _{t \rightarrow \infty} N(t)=\bar{N}
$$

In addition, The semiflow $\Phi$ has a compact attractor $\mathcal{A}$ of each bounded subset of $X^{+}$.

Proof. By summing the equations of (2.1) we easily obtain (3.1)-(3.2).

According to Theorem 2.33 in [28], we have to verify the following properties of $\Phi$ : (i) the point dissipative; (ii) eventually bounded on bounded sets of $X$; (iii) asymptotically smoothness. The conditions (i) and (ii) are satisfied by (3.1)-(3.2). For (iii), we use Theorem 2.46 in [28], by writing $\Phi$ as

$$
\Phi\left(t, \phi_{0}\right)=\Phi_{1}\left(t, \phi_{0}\right)+\Phi_{2}\left(t, \phi_{0}\right)
$$

with,

$$
\Phi_{1}\left(t, \phi_{0}\right):=\left(0, i_{1}(t, .), q_{1}(t, .), 0\right)
$$

and

$$
\Phi_{2}\left(t, \phi_{0}\right):=\left(S(t), i_{2}(t, .), q_{2}(t, .), R(t)\right)
$$

where

$$
\begin{gathered}
i_{1}(t, a)=\left\{\begin{array}{l}
0, \text { if } t>a, \\
i_{0}(a-t) \frac{\pi_{1}(t-a, a)}{\pi_{1}(t-a, a-t)}, \text { if } t \leq a,
\end{array}\right. \\
i_{2}(t, a)=\left\{\begin{array}{l}
i(t-a, 0) \pi_{1}(t-a, a), \text { if } t>a, \\
0, \text { if } t \leq a,
\end{array}\right. \\
q_{1}(t, a)=\left\{\begin{array}{l}
0, \text { if } t>a, \\
\frac{\pi_{2}(a)}{\pi_{2}(a-t)} q_{0}(a-t) \\
+i_{0}(a-t) \frac{\pi_{2}(a)}{\pi_{1}(t-a, a-t)} \int_{a-t}^{a} v(s) \psi(N(s+t-a)) \frac{\pi_{1}(t-a, s)}{\pi_{2}(s)} \mathrm{d} s, \text { if } t \leq a,
\end{array}\right.
\end{gathered}
$$

and

$$
q_{2}(t, a)=\left\{\begin{array}{l}
i(t-a, 0) \int_{0}^{a} v(s) \psi(N(s+t-a)) \frac{\pi_{2}(a)}{\pi_{2}(s)} \pi_{1}(t-a, s) \mathrm{d} s, \text { if } t>a \\
0, \text { if } t \leq a
\end{array}\right.
$$

Let $\mathbf{C}$ be a non-empty closed bounded set of initial conditions of $X$. By Theorem 3.1, we have the estimate 


$$
N(t) \leq \max \left(S_{0}+\left\|i_{0}\right\|_{1}+\left\|q_{0}\right\|_{1}+r_{0}, \bar{N}\right) .
$$

We put

$$
\begin{gathered}
c_{1}:=\sup \left\{S_{0}+\left\|i_{0}\right\|_{1}+\left\|q_{0}\right\|_{1}+r_{0}, \phi_{0} \in \mathbf{C}\right\}, \\
c_{2}:=\max \left\{c_{1}, \bar{N}\right\} .
\end{gathered}
$$

First we claim that, $\left\|\Phi_{1}\right\|_{1}$ tends to 0 as $t$ tends to 0 uniformly on $\mathbf{C}$. In fact,

$$
\begin{aligned}
& \left\|\Phi_{1}\right\|_{1}=\int_{0}^{\infty}\left|i_{1}(t, a)\right| \mathrm{d} a+\int_{0}^{\infty}\left|q_{1}(t, a)\right| \mathrm{d} a \\
& =\int_{0}^{\infty} \frac{\pi_{1}(-a, a+t)}{\pi_{1}(-a, a)} i_{0}(a) \mathrm{d} a+\int_{0}^{\infty} \frac{\pi_{2}(a+t)}{\pi_{2}(a)} q_{0}(a) \mathrm{d} a \\
& +\int_{0}^{\infty} \frac{\pi_{2}(a+t)}{\pi_{1}(-a, a)} i_{0}(a) \int_{0}^{t}\left(v(s+a) \psi(N(s)) \frac{\pi_{1}(-a, a+s)}{\pi_{2}(a+s)} \mathrm{d} s \mathrm{~d} a\right. \\
& =\int_{0}^{\infty} \exp \left\{-\int_{a}^{a+t}(\mu+v(s) \psi(N(s-a)) \mathrm{d} s\} i_{0}(a) \mathrm{d} a\right. \\
& +\int_{0}^{\infty} \exp \left\{-\int_{a}^{a+t}(\mu+\phi(s)) \mathrm{d} s\right\} q_{0}(a) \mathrm{d} a \\
& +\int_{0}^{\infty} i_{0}(a) \int_{0}^{t} v(s+a) \psi(N(s)) \frac{\pi_{2}(a+t)}{\pi_{2}(a+s)} \times \frac{\pi_{1}(-a, a+s)}{\pi_{1}(-a, a)} \mathrm{d} s \mathrm{~d} a \\
& =\exp (-\mu t)\left[\int _ { 0 } ^ { \infty } \operatorname { e x p } \left(-\int_{a}^{a+t} v(s) \psi(N(s-a)) i_{0}(a) e^{-\mu a} \mathrm{~d} a\right.\right. \\
& +\int_{0}^{\infty} \exp \left(-\int_{a}^{a+t} \phi(s) \mathrm{d} s\right) q_{0}(a) \mathrm{d} a+\int_{0}^{\infty} \int_{0}^{t} v(s+a) \psi(N(s)) \exp \left(-\int_{a+s}^{a+t} \phi(r) \mathrm{d} r\right) \\
& \left.\times \exp \left(-\int_{a}^{a+s} v(r) \psi(N(r-a)) \mathrm{d} r\right) e^{-\mu s} \mathrm{~d} s i_{0}(a) \mathrm{d} a\right] \\
& \leq \exp (-\mu t)\left\{2\left\|i_{0}\right\|_{1}+\left\|q_{0}\right\|_{1}\right\} \\
& \leq 3 c_{2} \exp (-\mu t) .
\end{aligned}
$$

We now claim that $\overline{\Phi_{2}(t, \mathbf{C})}$ is compact. To prove it, we need to verify condition (iii) of Theorem B.2. in [28]

$$
\begin{aligned}
I(h) & =\int_{0}^{t-h}\left(i_{2}(t, a+h)-i_{2}(t, a)\right) \mathrm{d} a+\int_{t-h}^{t}\left(i_{2}(t, a+h)-i_{2}(t, a)\right) \mathrm{d} a \\
& =\int_{0}^{t-h} \pi_{1}(t-a-h, a+h)(S(t-a-h) J(t-a-h)+\delta R(t-a-h)) \\
& -\pi_{1}(t-a, a)[S(t-a) J(t-a)+\delta R(t-a)] \mathrm{d} a \\
& -\int_{t-h}^{t} \pi_{1}(t-a, a)[S(t-a) J(t-a)+\delta R(t-a)] \mathrm{d} a .
\end{aligned}
$$


Since the last term tends to 0 as $h$ tends to 0 , we focus on the first integral

$$
\begin{aligned}
I_{1}(h) & =\int_{0}^{t-h} \pi_{1}(t-a-h, a+h)[S(t-a-h) J(t-a-h)+\delta R(t-a-h)] \\
& -\pi_{1}(t-a, a)[S(t-a) J(t-a)+\delta R(t-a)] \mathrm{d} a \\
& =\int_{0}^{t-h} \pi_{1}(t-a, a)[S(t-a-h) J(t-a-h) \\
& +\delta R(t-a-h)-S(t-a) J(t-a)-\delta R(t-a)] \mathrm{d} a \\
& +\int_{0}^{t-h}[S(t-a) J(t-a)+\delta R(t-a)]\left[\pi_{1}(t-a-h, a+h)-\pi_{1}(t-a, a)\right] \mathrm{d} a
\end{aligned}
$$

By subtracting and adding functions we obtain

$$
\begin{aligned}
& =\int_{0}^{t-h} \pi_{1}(t-a, a)[S(t-a-h) J(t-a-h)-S(t-a) J(t-a)] \mathrm{d} a \\
& +\delta \int_{0}^{t-h} \pi_{1}(t-a, a)[R(t-a-h)-R(t-a)] \mathrm{d} a+\int_{0}^{t-h}[S(t-a) J(t-a)+\delta R(t-a)] \\
& \times \quad\left[\pi_{1}(t-a-h, a+h)-\pi_{1}(t-a, a)\right] \mathrm{d} a \\
& =\int_{0}^{t-h} \pi_{1}(t-a, a)[S(t-a-h)-S(t-a)] J(t-a-h) \mathrm{d} a \\
& +\int_{0}^{t-h} \pi_{1}(t-a, a)[J(t-a-h)-J(t-a)] S(t-a) \mathrm{d} a \\
& +\delta \int_{0}^{t-h} \pi_{1}(t-a, a)[R(t-a-h)-R(t-a)] \mathrm{d} a \\
& +\quad\left(\|\beta\|_{\infty} c_{2}^{2}+\delta c_{2}\right) \int_{0}^{t-h}\left[\pi_{1}(t-a-h, a+h)-\pi_{1}(t-a, a)\right] \mathrm{d} a .
\end{aligned}
$$

By the continuity of $\pi_{1}$ with respect to $t$ and $a$ and thanks to the mean value theorem, the first, the third and the last terms tend to 0 as $h$ tends to 0 , since we have the following estimates on $S$ and $R$,

$$
\begin{aligned}
& \left|S^{\prime}(t)\right| \leq A+\mu c_{2}+\|\beta\|_{\infty} c_{2}^{2}, \\
& \left|R^{\prime}(t)\right| \leq\|\phi\|_{\infty} c_{2} .
\end{aligned}
$$

So, since $\pi_{1} \leq 1$ and $S(t) \leq c_{2}$ we have

$$
\left|I_{1}(h)\right| \leq\left|I_{2}(h)\right|=c_{2} \int_{0}^{t-h}|J(t-a-h)-J(t-a)| \mathrm{d} a .
$$


Set

$$
J(t)=\bar{J}_{1}(t)+\bar{J}_{2}(t)
$$

with

$$
\begin{aligned}
\bar{J}_{1}(t) & =\int_{0}^{t} \beta(a) \pi_{1}(t-a, a)[S(t-a) J(t-a)+\delta R(t-a)] \mathrm{d} a \\
& =\int_{0}^{t} \beta(t-\sigma) \pi_{1}(\sigma, t-\sigma)[S(\sigma) J(\sigma)+\delta R(\sigma)] \mathrm{d} \sigma,
\end{aligned}
$$

and

$$
\begin{aligned}
\bar{J}_{2}(t) & :=\int_{t}^{\infty} \beta(a) \frac{\pi_{1}(t-a, a)}{\pi_{1}(t-a, a-t)} i_{0}(a-t) \mathrm{d} a \\
& =\int_{0}^{\infty} \beta(a+t) \frac{\pi_{1}(-a, a+t)}{\pi_{1}(-a, a)} i_{0}(a) \mathrm{d} a .
\end{aligned}
$$

Thus, for $r, h>0$, we have

$$
|J(r+h)-J(r)| \leq\left|\bar{J}_{1}(r+h)-\bar{J}_{1}(r)\right|+\left|\bar{J}_{2}(r+h)-\bar{J}_{2}(r)\right|
$$

We focus on each term separately,

$$
\begin{aligned}
\left|\bar{J}_{1}(r+h)-\bar{J}_{1}(r)\right| & \leq \int_{r}^{r+h} \beta(r+h-s) \pi_{1}(s, r+h-s)[S(s) J(s)+\delta R(s)] \mathrm{d} s, \\
& +\int_{0}^{r}\left|\beta(r+h-s) \pi_{1}(s, r+h-s)-\beta(r-s) \pi_{1}(s, r-s)\right| \\
& \times[S(s) J(s)+\delta R(s)] \mathrm{d} s, \\
& \leq h\left[\|\beta\|_{\infty}^{2} c_{2}^{2}+\delta\|\beta\|_{\infty} c_{2}\right]+\left[\|\beta\|_{\infty} c_{2}^{2}+\delta c_{2}\right] \\
& \times\left\{\int_{0}^{r} \beta(r+h-s)\left|\pi_{1}(s, r+h-s)-\pi_{1}(s, r-s)\right| \mathrm{d} s\right\} \\
& +\int_{0}^{r} \pi_{1}(s, r-s)|\beta(r+h-s)-\beta(r-s)| \mathrm{d} s,
\end{aligned}
$$


which tends to 0 as $h$ tends to zero, uniformly on $\mathbf{C}$, since $\beta$ and $\pi_{1}$ are bounded and uniformly continuous.

$$
\begin{aligned}
\left|\bar{J}_{2}(r+h)-\bar{J}_{2}(r)\right| & =\mid \int_{0}^{\infty} \beta(a+r+h) \frac{\pi_{1}(-a, a+r+h)}{\pi_{1}(-a, a)} i_{0}(a) \mathrm{d} a \\
& -\int_{0}^{\infty} \beta(a+r) \frac{\pi_{1}(-a, a+r)}{\pi_{1}(-a, a)} i_{0}(a) \mathrm{d} a \mid \\
& \leq \int_{0}^{\infty} \beta(a+r+h) i_{0}(a)\left|\frac{\pi_{1}(-a, a+r+h)}{\pi_{1}(-a, a)}-\frac{\pi_{1}(-a, a+r)}{\pi_{1}(-a, a)}\right| \mathrm{d} a \\
& +\int_{0}^{\infty} \frac{\pi_{1}(-a, a+r)}{\pi_{1}(-a, a)}|\beta(a+r+h)-\beta(a+r)| i_{0}(a) \mathrm{d} a .
\end{aligned}
$$

By the same arguments as above, this difference tends to 0 as $h$ tends to 0 , uniformly on $\mathbf{C}$. This finishes the proof.

\subsection{Total trajectories}

Let $\mathcal{U}=(S(t), i(t,),. q(t,),. R(t))$ and $\mathcal{U}(t+r)=\Phi(t, \mathcal{U}(r))$.

Set $\left(S_{r}(t), i_{r}(t, a), q_{r}(t, a), R_{r}(t)\right)=(S(t+r), i(t+r, a), q(t+r, a), R(t+r))$ and $J_{r}(t)=J(t+r)$.

In view of (2.3) and (2.4) and the definition of the semiflow we have

$$
\begin{gathered}
S_{r}^{\prime}(t)=A-S_{r}(t) J_{r}(t)-\mu S_{r}(t), \quad S_{r}(0)=S(r), \\
i_{r}(t, a)=\left\{\begin{array}{l}
\left(S_{r}(t-a) J_{r}(t-a)+\delta R_{r}(t-a)\right) \pi_{1}(t-a, a), \quad \text { if } t>a, \\
i(r, a-t) \frac{\pi_{1}(t-a, a)}{\pi_{1}(t-a, a-t)}, \text { if } t<a,
\end{array}\right. \\
q_{r}(t, a)=\left\{\begin{array}{l}
i_{r}(t-a, 0) \int_{0}^{a} v(s) \psi\left(N_{r}(s+t-a)\right) \frac{\pi_{2}(a)}{\pi_{2}(s)} \pi_{1}(s+t-a, s) \mathrm{d} s, \text { if } t>a, \\
\frac{\pi_{2}(a)}{\pi_{2}(a-t)} q(r, a-t) \\
+i(r, a-t) \frac{\pi_{2}(a)}{\pi_{1}(t-a, a-t)} \int_{a-t}^{a} v(s) \psi\left(N_{r}(s+t-a)\right) \frac{\pi_{1}(t-a, s)}{\pi_{2}(s)} \mathrm{d} s, \text { if } t<a,
\end{array}\right.
\end{gathered}
$$

and

$$
\left\{\begin{array}{l}
R_{r}^{\prime}(t)=\int_{0}^{\infty} \phi(a) q_{r}(t, a) \mathrm{d} a-(\mu+\delta) R_{r}(t), \\
N_{r}^{\prime}(t)=A-\mu N_{r}(t) .
\end{array}\right.
$$


Set $t=s-r$ with $s \geq r$ then

$$
\begin{gathered}
S^{\prime}(s)=A-S(s) J(s)-\mu S(s), \\
i(s, a)=\left\{\begin{array}{l}
(S(s-a) J(s-a)+\delta R(s-a)) \pi_{1}(s-r-a, a) \text { if } s-r>a \\
i(r, a-s+r) \frac{\pi_{1}(s-r-a, a)}{\pi_{1}(s-r-a, a-s+r)} \text { if } s-r<a
\end{array}\right. \\
q(s, a)=\left\{\begin{array}{l}
i(s-a, 0) \int_{0}^{a} v(\sigma) \psi(N(\sigma+s-r-a)) \frac{\pi_{2}(a)}{\pi_{2}(\sigma)} \pi_{1}(s-r-a, \sigma) \mathrm{d} \sigma, \text { if } s-r>a, \\
\frac{\pi_{2}(a)}{\pi_{2}(a-s+r)} q(r, a-s+r) \\
+i(r, a-s+r) \frac{\pi_{2}(a)}{\pi_{1}(s-r-a, a-s+r)} \\
\times \int_{a-s+r}^{a} v(s) \psi(N(\sigma+s-r-a)) \frac{\pi_{1}(s-r-a, \sigma)}{\pi_{2}(\sigma)} \mathrm{d} \sigma, \quad \text { if } s-r<a,
\end{array}\right.
\end{gathered}
$$

and

$$
\left\{\begin{array}{l}
R^{\prime}(s)=\int_{0}^{\infty} \phi(a) q(s, a) \mathrm{d} a-(\mu+\delta) R(s), \\
N^{\prime}(s)=A-\mu N(s) .
\end{array}\right.
$$

Now by straightforward computations we obtain

$$
N(s)=N(r) e^{\mu(r-s)}+\frac{A}{\mu}\left(1-e^{\mu(r-s)}\right) \text { for } s \geq r .
$$

Next by letting $r \rightarrow-\infty$ we get

$$
N(s)=\bar{N} \quad \text { for all } \quad s \in \mathbb{R} .
$$

This, together with $r \rightarrow-\infty$, gives the following total trajectory, for all $t \in \mathbb{R}$,

$$
\left\{\begin{array}{l}
S^{\prime}(t)=A-S(t) J(t)-\mu S(t), \\
i(t, a)=i(t-a, 0) \bar{\pi}_{1}(a), \\
q(t, a)=i(t-a, 0) \pi_{2}(a) \int_{0}^{a} v(\sigma) \psi(\bar{N}) \frac{\bar{\pi}_{1}(\sigma)}{\pi_{2}(\sigma)} \mathrm{d} \sigma, \\
R^{\prime}(t)=\int_{0}^{\infty} \phi(a) q(t, a) \mathrm{d} a-(\mu+\delta) R(t), \\
i(t, 0)=S(t) J(t)+\delta R(t),
\end{array}\right.
$$


with

$$
\left\{\begin{array}{l}
\bar{\pi}_{1}(a)=\exp \left(-\int_{0}^{a}(\mu+v(s) \psi(\bar{N})) \mathrm{d} s\right) \\
\pi_{2}(a):=\exp \left(-\int_{0}^{a}(\mu+\phi(s)) \mathrm{d} s\right)
\end{array}\right.
$$

Lemma 3.2. For all $\phi_{0} \in \mathcal{A}$ we have

$$
S(t)+\int_{0}^{\infty} i(t, a) \mathrm{d} a+\int_{0}^{\infty} q(t, a) \mathrm{d} a+R(t)=\bar{N}
$$

and

$$
S(t) \geq \frac{A}{\mu+\|\beta\| \bar{N}}
$$

for all $t \in \mathbb{R}$.

Proof. We set $I(t)=\int_{0}^{\infty} i(t, a) \mathrm{d} a$ and $Q(t)=\int_{0}^{\infty} q(t, a) \mathrm{d} a$. Using the expression of $i$ and $q$ in (3.4) and after a change of variable we get

$$
I(t)=\int_{-\infty}^{t} i(s, 0) \bar{\pi}_{1}(t-s) \mathrm{d} s
$$

and

$$
Q(t)=\int_{-\infty}^{t} i(s, 0) \pi_{2}(t-s) \int_{0}^{t-s} v(\sigma) \psi(\bar{N}) \frac{\bar{\pi}_{1}(\sigma)}{\pi_{2}(\sigma)} \mathrm{d} \sigma \mathrm{d} s
$$

The functions, $I$ and $Q$ satisfy the following equations

$$
I^{\prime}(t)=i(t, 0)-\mu I(t)-\int_{-\infty}^{t} i(s, 0) v(t-s) \psi(\bar{N}) \bar{\pi}_{1}(t-s) \mathrm{d} s
$$

and

$$
\begin{aligned}
Q^{\prime}(t) & =-\mu Q(t)-\int_{-\infty}^{t} i(s, 0) \phi(t-s) \pi_{2}(t-s) \int_{0}^{t-s} v(\sigma) \psi(\bar{N}) \frac{\bar{\pi}_{1}(\sigma)}{\pi_{2}(\sigma)} \mathrm{d} \sigma \mathrm{d} s \\
& +\int_{-\infty}^{t} i(s, 0) \bar{\pi}_{1}(t-s) v(t-s) \psi(\bar{N}) \mathrm{d} s
\end{aligned}
$$

Substituting $q$ by its expression and after a change of variable we have

$$
R^{\prime}(t)=\int_{-\infty}^{t} \phi(t-s) i(s, 0) \pi_{2}(t-s) \int_{0}^{t-s} v(\sigma) \psi(\bar{N}) \frac{\bar{\pi}_{1}(\sigma)}{\pi_{2}(\sigma)} \mathrm{d} \sigma \mathrm{d} s-(\mu+\delta) R(t) .
$$

Now, for $N(t)=S(t)+I(t)+Q(t)+R(t)$ we have

$$
N^{\prime}(t)=A-\mu N(t)
$$


Finally, using the same computation as for the total trajectory of $N$, stated above, we obtain $N(t)=\bar{N}$ for all $t \in \mathbb{R}$. Furthermore, it is easy to observe that

$$
S^{\prime}(t) \geq A-\left(\mu+\|\beta\|_{\infty} \bar{N}\right) S(t)
$$

and thus, by a straightforward computation,

$$
S(t) \geq \frac{A}{\mu+\|\beta\|_{\infty} \bar{N}}
$$

for all $t \in \mathbb{R}$.

We define the epidemic reproduction number for (2.1) as

$$
R_{0}=\int_{0}^{\infty} \bar{N} \beta(s) \bar{\pi}_{1}(s) \mathrm{d} s+\frac{\delta}{\delta+\mu} \psi(\bar{N}) \int_{0}^{\infty} v(s) \frac{\bar{\pi}_{1}(s)}{\pi_{2}(s)} \int_{s}^{\infty} \phi(\sigma) \pi_{2}(\sigma) \mathrm{d} \sigma \mathrm{d} s .
$$

Remark 3.3. By Fubini's Theorem we also have

$$
R_{0}=\int_{0}^{\infty} \bar{N} \beta(s) \bar{\pi}_{1}(s) \mathrm{d} s+\frac{\delta}{\delta+\mu} \psi(\bar{N}) \int_{0}^{\infty} \phi(\sigma) \pi_{2}(\sigma) \int_{0}^{\sigma} v(s) \frac{\bar{\pi}_{1}(s)}{\pi_{2}(s)} \mathrm{d} s \mathrm{~d} \sigma .
$$

\section{Global Stability of the Disease Free EQUilibrium}

Theorem 4.1. If $R_{0} \leq 1$, then $E_{0}=(\bar{N}, 0,0,0)$ is globally asymptotically stable.

Proof. Let $\phi_{0} \in \mathcal{A}$. Since the compact attractor $\mathcal{A}$ is invariant, then there exists, a total trajectory $\Psi: \mathbb{R} \rightarrow \mathcal{A}$ such that $\Psi(t)=(S(t), i(t,),. q(t,),. R(t))$ solution of (3.4) passing through $\phi_{0}$. Set

$$
k(a)=\frac{\delta}{\delta+\mu} \int_{a}^{\infty} \phi(\sigma) \frac{\pi_{2}(\sigma)}{\pi_{2}(a)} \mathrm{d} \sigma
$$

and

$$
h(a)=\frac{1}{\bar{\pi}_{1}(a)}\left(1-\int_{0}^{a}\left(\bar{N} \beta(\sigma) \bar{\pi}_{1}(\sigma)+\frac{\delta}{\delta+\mu} \psi(\bar{N}) v(\sigma) \frac{\bar{\pi}_{1}(\sigma)}{\pi_{2}(\sigma)} \int_{\sigma}^{\infty} \phi(\xi) \pi_{2}(\xi) \mathrm{d} \xi\right) \mathrm{d} \sigma\right) .
$$

Remark 4.2. Observe that $h(a) \geq 0$ for $R_{0} \leq 1$.

We consider the following functional

$$
V(\Psi(t))=F(S)+\int_{0}^{\infty} h(a) i(t, a) \mathrm{d} a+\int_{0}^{\infty} k(a) q(t, a) \mathrm{d} a+\frac{\delta}{\delta+\mu} R(t),
$$

with $F(S)=S-\bar{N} \ln \frac{S}{\bar{N}}-\bar{N}$ and $\bar{N}=\frac{A}{\mu}$. Set

$$
I_{1}(t)=\int_{0}^{\infty} h(a) i(t, a) \mathrm{d} a
$$


and

$$
Q_{1}(t)=\int_{0}^{\infty} k(a) q(t, a) \mathrm{d} a
$$

Differentiating $F(S)$ along the solution of (3.4) we obtain

$$
\frac{\mathrm{d}}{\mathrm{d} t} F(S(t))=\mu\left(1-\frac{\bar{N}}{S}\right)(\bar{N}-S)-S(t) J(t)+\bar{N} J(t)
$$

Next, from the expression of $i$ in (3.4) we have

$$
I_{1}(t)=\int_{0}^{\infty} P_{1}(a) B(t-a) \mathrm{d} a
$$

with $B(t)=i(t, 0)$ and $P_{1}(a)=\bar{\pi}_{1}(a) h(a)$. Following the same arguments as in the proof of Lemma 9.18 in [28] we can show that $I_{1}$ is absolutely continuous and

$$
I_{1}^{\prime}(t)=P_{1}(0) B(t)+\int_{0}^{\infty} P_{1}^{\prime}(a) B(t-a) \mathrm{d} a .
$$

By expanding $P_{1}^{\prime}$ we obtain

$$
I_{1}^{\prime}(t)=B(t)-\int_{0}^{\infty}(\bar{N} \beta(a)+v(a) \psi(\bar{N}) k(a)) \bar{\pi}_{1}(a) B(t-a) \mathrm{d} a
$$

Similarly, we have

$$
Q_{1}(t)=\int_{0}^{\infty} P_{2}(a) B(t-a) \mathrm{d} a,
$$

with $P_{2}(a)=k(a) \pi_{2}(a) \int_{0}^{a} v(\sigma) \psi(\bar{N}) \frac{\bar{\pi}_{1}(\sigma)}{\pi_{2}(\sigma)} \mathrm{d} \sigma$, so

$$
Q_{1}^{\prime}(t)=\int_{0}^{\infty} P_{2}^{\prime}(a) B(t-a) \mathrm{d} a .
$$

By expanding $P_{2}^{\prime}$ we obtain

$$
Q_{1}^{\prime}(t)=\int_{0}^{\infty}\left(-\frac{\delta}{\delta+\mu} \phi(a) \pi_{2}(a) \int_{0}^{a} v(\sigma) \psi(\bar{N}) \frac{\bar{\pi}_{1}(a)}{\pi_{2}(\sigma)} \mathrm{d} \sigma+k(a) \bar{\pi}_{1}(a) v(a) \psi(\bar{N})\right) B(t-a) \mathrm{d} a .
$$


Now we compute the derivative of $V$ and by using the formulas in (3.4) we get

$$
\begin{aligned}
\frac{\mathrm{d}}{\mathrm{d} t} V(\Psi(t)) & =\mu\left(1-\frac{\bar{N}}{S}\right)(\bar{N}-S)-S(t) J(t)+\bar{N} \int_{0}^{\infty} \beta(a) \bar{\pi}_{1}(a) B(t-a) \mathrm{d} a \\
& +S(t) J(t)+\delta R(t)-\int_{0}^{\infty}(\bar{N} \beta(a)+v(a) \psi(\bar{N}) k(a)) \bar{\pi}_{1}(a) B(t-a) \mathrm{d} a \\
& +\int_{0}^{\infty}\left(-\frac{\delta}{\delta+\mu} \phi(a) \pi_{2}(a) \int_{0}^{a} v(\sigma) \psi(\bar{N}) \frac{\bar{\pi}_{1}(\sigma)}{\pi_{2}(\sigma)} \mathrm{d} \sigma+k(a) \bar{\pi}_{1}(a) v(a) \psi(\bar{N})\right) B(t-a) \mathrm{d} a \\
& +\frac{\delta}{\mu+\delta}\left(\int_{0}^{\infty} \phi(a) B(t-a) \pi_{2}(a) \int_{0}^{a} v(\sigma) \psi(\bar{N}) \frac{\bar{\pi}_{1}(\sigma)}{\pi_{2}(\sigma)} \mathrm{d} \sigma \mathrm{d} a-(\mu+\delta) R(t)\right) .
\end{aligned}
$$

By simplification

$$
\begin{aligned}
\frac{\mathrm{d}}{\mathrm{d} t} V(\Psi(t)) & =\mu\left(1-\frac{\bar{N}}{S}\right)(\bar{N}-S) \\
& -\int_{0}^{\infty} \frac{\delta}{\delta+\mu} \phi(a) \pi_{2}(a) \int_{0}^{a} v(\sigma) \psi(\bar{N}) \frac{\bar{\pi}_{1}(\sigma)}{\pi_{2}(\sigma)} \mathrm{d} \sigma B(t-a) \mathrm{d} a \\
& +\frac{\delta}{\mu+\delta} \int_{0}^{\infty} \phi(a) \pi_{2}(a) \int_{0}^{a} v(\sigma) \psi(\bar{N}) \frac{\bar{\pi}_{1}(\sigma)}{\pi_{2}(\sigma)} \mathrm{d} \sigma B(t-a) \mathrm{d} a \\
& =\mu\left(1-\frac{N}{S}\right)(\bar{N}-S) \leq 0 .
\end{aligned}
$$

Notice that $\frac{\mathrm{d}}{\mathrm{d} t} V(\Psi(t))=0$ implies that $S=\bar{N}$. Due to (3.6) we get $i=q=R=0$. Hence the largest invariant set with the property that $\frac{\mathrm{d}}{\mathrm{d} t} V(\Psi(t))=0$ is $\left\{E_{0}\right\}$ (LaSalle's invariance principle). Now since $\mathcal{A}$ is compact, the $\omega\left(\phi_{0}\right)$ and $\alpha\left(\phi_{0}\right)$ (omega and alpha limits sets respectively) are non empty, compact, invariant and attract $\psi(t)$ as $t \rightarrow \pm \infty$ respectively. Since $V(\Psi(t))$ is a decreasing function of $t, V$ is constant on the $\omega\left(\phi_{0}\right)$ and $\alpha\left(\phi_{0}\right)$ and thus $\omega\left(\phi_{0}\right)=\alpha\left(\phi_{0}\right)=\left\{E_{0}\right\}$. Consequently, $\lim _{t \rightarrow \pm \infty} \Psi(t)=E_{0}$ and

$$
\lim _{t \rightarrow-\infty} V(\Psi(t))=\lim _{t \rightarrow+\infty} V(\Psi(t))=E_{0}
$$

Finally we obtain $V(\Psi(t))=V\left(E_{0}\right)$ for all $t \in \mathbb{R}$. Since $\alpha\left(\phi_{0}\right)=\left\{E_{0}\right\}$ then $V(\Psi(t)) \leq V\left(E_{0}\right)$ for all $t \in \mathbb{R}$. Since $V$ achieves its minimum value at $E_{0}$ we conclude that $\Psi(t)=E_{0}$ for all $t \in \mathbb{R}$. In particular $\phi_{0}=E_{0}$. Therefore the attractor $\mathcal{A}$ is the singleton set formed by the disease free equilibrium $E_{0}$. By Theorem 2.39 in [28] the disease free is globally asymptotically stable.

\section{Existence, uniqueness And GLObal Stability of the Endemic EQUILIBRIUM}

We first begin by proving existence and uniqueness of the positive equilibrium provided $R_{0}>1$.

Lemma 5.1. Suppose that $R_{0}>1$ then there exists a unique positive equilibrium of (3.4). 
Proof. First the equilibrium problem satisfies

$$
\left\{\begin{array}{l}
A=S^{*} J^{*}+\mu S^{*} \\
i^{*}(a)=i^{*}(0) \bar{\pi}_{1}(a) \\
q^{*}(a)=i^{*}(0) \pi_{2}(a) \int_{0}^{a} v(\sigma) \psi(\bar{N}) \frac{\bar{\pi}_{1}(\sigma)}{\pi_{2}(\sigma)} \mathrm{d} \sigma \\
R^{*}=\frac{1}{\mu+\delta} \int_{0}^{\infty} \phi(a) q^{*}(a) \mathrm{d} a \\
i^{*}(0)=S^{*} J^{*}+\delta R^{*} \text { and } J^{*}=i^{*}(0) \int_{0}^{\infty} \beta(a) \bar{\pi}_{1}(a) \mathrm{d} a
\end{array}\right.
$$

Set $F(a)=\pi_{2}(a) \int_{0}^{a} v(\sigma) \psi(\bar{N}) \frac{\bar{\pi}_{1}(\sigma)}{\pi_{2}(\sigma)} \mathrm{d} \sigma$. Then, combining the third, fourth and the last equation of (5.1) we obtain

$$
i^{*}(0)=S^{*} i^{*}(0) \int_{0}^{\infty} \beta(a) \bar{\pi}_{1}(a) \mathrm{d} a+i^{*}(0) \frac{\delta}{\mu+\delta} \int_{0}^{\infty} \phi(a) F(a) \mathrm{d} a
$$

so, for $i^{*}(0) \neq 0$ we have

$$
S^{*} \int_{0}^{\infty} \beta(a) \bar{\pi}_{1}(a) \mathrm{d} a+\frac{\delta}{\mu+\delta} \int_{0}^{\infty} \phi(a) F(a) \mathrm{d} a=1 .
$$

Now using the first equation of (5.1) and combining it with (5.2) we get

$$
A \int_{0}^{\infty} \beta(a) \bar{\pi}_{1}(a) \mathrm{d} a+\left(\mu+J^{*}\right) \frac{\delta}{\mu+\delta} \int_{0}^{\infty} \phi(a) F(a) \mathrm{d} a=\mu+J^{*}
$$

Thus,

$$
J^{*}\left(1-\frac{\delta}{\mu+\delta} \int_{0}^{\infty} \phi(a) F(a) \mathrm{d} a\right)=A \int_{0}^{\infty} \beta(a) \bar{\pi}_{1}(a) \mathrm{d} a+\mu\left(\frac{\delta}{\mu+\delta} \int_{0}^{\infty} \phi(a) F(a) \mathrm{d} a-1\right),
$$

therefore

$$
\begin{aligned}
J^{*}\left(1-\frac{\delta}{\mu+\delta} \int_{0}^{\infty} \phi(a) F(a) \mathrm{d} a\right) & =\mu\left(\bar{N} \int_{0}^{\infty} \beta(a) \bar{\pi}_{1}(a) \mathrm{d} a+\frac{\delta}{\mu+\delta} \int_{0}^{\infty} \phi(a) F(a) \mathrm{d} a-1\right), \\
& =\mu\left(R_{0}-1\right) .
\end{aligned}
$$

Note that $\frac{\delta}{\mu+\delta} \int_{0}^{\infty} \phi(a) F(a) \mathrm{d} a<1$. Indeed, it is easily to see that $F(a) \leq 1$ for all $a \geq 0$ then, $\frac{\delta}{\mu+\delta} \int_{0}^{\infty} \phi(a) F(a) \mathrm{d} a \leq \frac{\delta}{\mu+\delta} \int_{0}^{\infty} \phi(a) \mathrm{d} a<1$. This, together with $R_{0}>1$, leads to the existence of $J^{*}>0$, and thus the existence of $i^{*}(0)$. Finally, according to (5.1) we conclude the existence and the uniqueness of the positive equilibrium $\left(S^{*}, i^{*}(),. q^{*}(),. R^{*}\right)$. The lemma is proved. 


\subsection{Uniform persistence of the system}

Let $\rho: X^{+} \rightarrow \mathbb{R}$ be a function defined by

$$
\rho\left(\Phi(t) \phi_{0}\right)=i(t, 0):=B(t), \quad t \geq 0, \quad \phi_{0} \in X^{+}
$$

and

$$
\Omega_{0}=\left\{\phi_{0} \in X^{+}, \quad \rho\left(\phi_{0}\right)>0\right\}
$$

We first prove the following Lemma.

Lemma 5.2. If $\rho(\mathcal{U}(t))=0$ for all $t \leq 0$, then $\rho(\mathcal{U}(t))=0$ for all $t>0$.

Proof. Suppose that $\rho(\mathcal{U}(t))=0$ for all $t \leq 0$, then, $J(t)=0$ and $R(t)=0$ for all $t \leq 0$. Further, from (3.4),

$$
B(t)=S(t) \int_{0}^{t} \beta(a) \bar{\pi}_{1}(a) B(t-a) \mathrm{d} a+\delta R(t) .
$$

By solving the equation of $R$ in (3.4) we obtain

$$
R(t)=r_{0} e^{-(\mu+\delta) t}+\int_{0}^{t} e^{-(\mu+\delta)(t-\sigma)} \int_{0}^{\sigma} \phi(a) B(\sigma-a) F(a) \mathrm{d} a \mathrm{~d} \sigma,
$$

with $F(a)=\pi_{2}(a) \int_{0}^{a} v(\sigma) \psi(\bar{N}) \frac{\bar{\pi}_{1}(\sigma)}{\pi_{2}(\sigma)} \mathrm{d} \sigma$. Substituting the expression of $R$ in (5.3) and using the fact that $R(0)=0$, we have

$$
B(t)=S(t) \int_{0}^{t} \beta(a) \bar{\pi}_{1}(a) B(t-a) \mathrm{d} a+\delta \int_{0}^{t} e^{-(\mu+\delta)(t-\sigma)} \int_{0}^{\sigma} \phi(a) B(\sigma-a) F(a) \mathrm{d} a \mathrm{~d} \sigma,
$$

Since $F$ is a bounded function, then from Fubini's theorem,

$$
\begin{aligned}
B(t) & \leq \bar{N}\|\beta\|_{\infty} \int_{0}^{t} B(a) \mathrm{d} a+\delta \int_{0}^{t} B(a) \int_{a}^{t} e^{-(\mu+\delta)(t-\sigma)} \phi(\sigma-a) F(\sigma-a) \mathrm{d} \sigma \mathrm{d} a \\
& \leq\left(\bar{N}\|\beta\|_{\infty}+\frac{\delta\|\phi F\|_{\infty}}{\mu+\delta}\right) \int_{0}^{t} B(a) \mathrm{d} a
\end{aligned}
$$

Gronwall's inequality leads to $B(t)=0$ for all $t \in \mathbb{R}$.

Lemma 5.3. Assume that either $\beta$ or $\phi$ is not equal to zero almost everywhere. Then, either $\rho(\mathcal{U}()$.$) is identically$ null in $\mathbb{R}$ or $\rho(\mathcal{U}()$.$) is positive everywhere on \mathbb{R}$.

Proof. From the previous Lemma, by an appropriate shift, for each $r \in \mathbb{R}$ such that $\rho(\mathcal{U}(t))=0$ for all $t \leq r$ implies that $\rho(\mathcal{U}(t))=0$ for all $t \geq r$. Thus, either $\rho(\mathcal{U}()$.$) is identically null or there exists a sequence t_{j} \rightarrow-\infty$ 
as $j \rightarrow+\infty$ with $\rho\left(\mathcal{U}\left(t_{j}\right)\right)>0$. Assume the second. Let $B_{j}(t)=B\left(t+t_{j}\right)$. By Lemma 3.2 , we have

$$
\begin{aligned}
B_{j}(t) & \geq \frac{A}{\mu+\|\beta\| \bar{N}} \int_{0}^{t} \beta(a) \bar{\pi}_{1}(a) B_{j}(t-a) \mathrm{d} a \\
& +\delta \int_{0}^{t} e^{-(\mu+\delta)(t-\sigma)} \int_{0}^{\sigma} \phi(a) B_{j}(\sigma-a) F(a) \mathrm{d} a \mathrm{~d} \sigma+\hat{B}_{j}(t)
\end{aligned}
$$

with $\hat{B}_{j}(0)=B\left(t_{j}\right)>0$. Using Fubini's theorem,

$$
\begin{aligned}
B_{j}(t) & \geq \frac{A}{\mu+\|\beta\| \bar{N}} \int_{0}^{t} \beta(a) \bar{\pi}_{1}(a) B_{j}(t-a) \mathrm{d} a \\
& +\delta \int_{0}^{t} B_{j}(a) \int_{a}^{t} e^{-(\mu+\delta)(t-\sigma)} \phi(\sigma-a) F(\sigma-a) \mathrm{d} \sigma \mathrm{d} a+\hat{B}_{j}(t), \\
& \geq \int_{0}^{t}\left(\frac{A}{\mu+\|\beta\| \bar{N}} \beta(t-a) \pi_{1}(t-a)+\delta \xi(t-a)\right) B_{j}(a) \mathrm{d} a+\hat{B}_{j}(t),
\end{aligned}
$$

where $\xi(t)=\int_{0}^{t} e^{-(\mu+\delta)(t-\sigma)} \phi(\sigma) F(\sigma) \mathrm{d} \sigma$. Note that $\hat{B}_{j}$ is continuous at zero and $\hat{B}_{j}(0)>0$. Therefore, by Corollary B.6 in [28], we see that there exists $b>0$ such that $\rho\left(\mathcal{U}_{j}(t)\right)>0$ for all $t>b$. Thus $\rho(\mathcal{U}(t))>0$ for all $t>b+t_{j}$. By passing to the limit as $j \rightarrow \infty$ we prove that $\rho(\mathcal{U}(t))>0$ for all $t \in \mathbb{R}$. Consequently $\rho(\mathcal{U}()$. is positive on $\mathbb{R}$.

The following lemma states the uniform persistence of the semiflow.

Lemma 5.4. Assume that $R_{0}>1$. Then, system (2.1) is uniformly strongly persistent for non-trivial initial data, that is, there exists $\epsilon>0$ such that $\liminf _{t \rightarrow \infty} \rho\left(\Phi(t) \phi_{0}\right) \geq \epsilon$ for all $\phi_{0} \in \Omega_{0}$.

Proof. First, by Lemmas 5.2, 5.3, we can apply Theorem 5.2 in [28] to conclude that the weak uniform persistence implies the strong uniform persistence.

Suppose now, by contradiction, that the semiflow $\Phi$ is not weakly persistent, that is,

$$
\limsup _{t \rightarrow \infty} \rho\left(\Phi(t) \phi_{0}\right)=0
$$

This, with (2.3)-(2.4), implies that $\lim _{t \rightarrow \infty} i(t,)=.\lim _{t \rightarrow \infty} q(t,)=$.0 and $\lim _{t \rightarrow \infty} R(t)=\lim _{t \rightarrow \infty} J(t)=0$. Thus, there exists $T>0$ such that $J(t) \leq \epsilon$ for all $\epsilon>0$ and $t \geq T$. Hence, from the equation of $S$ in (2.1) we get $S(t) \geq \bar{N}-\varepsilon$ for all $t \geq T$. In the other hand, since $N(t) \rightarrow \bar{N}$ as $t \rightarrow \infty$ and $\psi$ is a continuous function then, there exists $T>0$ such that $\psi(\bar{N})-\varepsilon \leq \psi(N(t)) \leq \psi(\bar{N})+\varepsilon$ for all $t \geq T$. 
We introduce the following problem

$$
\left\{\begin{array}{l}
\frac{\partial \tilde{i}(t, a)}{\partial t}+\frac{\partial \tilde{i}(t, a)}{\partial a}=-(\mu+v(a)(\psi(\bar{N})+\varepsilon)) \tilde{i}(t, a) \\
\frac{\partial \tilde{q}(t, a)}{\partial t}+\frac{\partial \tilde{q}(t, a)}{\partial a}=v(a)(\psi(\bar{N})-\varepsilon) \tilde{i}(t, a)-(\mu+\phi(a)) \tilde{q}(t, a) \\
\tilde{R}^{\prime}(t)=\int_{0}^{\infty} \phi(a) \tilde{q}(t, a) \mathrm{d} a-(\mu+\delta) \tilde{R}(t) \\
\tilde{i}(t, 0)=(\bar{N}-\varepsilon) \tilde{J}(t)+\delta \tilde{R}(t) \\
\tilde{q}(t, 0)=0 \\
\tilde{J}(t)=\int_{0}^{\infty} \beta(a) \tilde{i}(t, a) \mathrm{d} a
\end{array}\right.
$$

with the same initial data as in (2.2). From a comparison principle we can see that there exists $T>0$ such that $\tilde{i}(t,.) \leq i(t,),. \tilde{q}(t,.) \leq q(t,$.$) and \tilde{R}(t) \leq R(t)$ for all $t \geq T$.

Now, since $R_{0}>1$ then for $\varepsilon>0$ so small, there exists $\lambda_{\varepsilon}>0$ such that

$$
\begin{aligned}
& \int_{0}^{\infty}[(\bar{N}-\varepsilon) \beta(s) \\
+ & \left.\frac{\delta}{\mu+\delta+\varepsilon} v(s)(\psi(\bar{N})-\varepsilon) \int_{s}^{\infty} \phi(\sigma) \frac{\pi_{2}(\sigma)}{\pi_{2}(s)} e^{-\varepsilon(\sigma-s)} \mathrm{d} \sigma\right] \bar{\pi}_{1}(s) e^{-\int_{0}^{s}\left(\lambda_{\varepsilon}+\varepsilon v(\xi)\right) \mathrm{d} \xi} \mathrm{d} s=1 .
\end{aligned}
$$

Set,

$$
k_{\varepsilon}(a)=\frac{\delta}{\mu+\delta+\varepsilon} \int_{a}^{\infty} \phi(s) \frac{\pi_{2}(s)}{\pi_{2}(a)} e^{-\varepsilon(s-a)} \mathrm{d} s,
$$

and

$$
\begin{aligned}
h_{\varepsilon}(a) \bar{\pi}_{1}(a) & =1-\int_{0}^{a}[(\bar{N}-\varepsilon) \beta(s) \\
& \left.+\frac{\delta}{\mu+\delta+\varepsilon} v(s)(\psi(\bar{N})-\varepsilon) \int_{s}^{\infty} \phi(\sigma) \frac{\pi_{2}(\sigma)}{\pi_{2}(s)} e^{-\varepsilon(\sigma-s)} \mathrm{d} \sigma\right] \bar{\pi}_{1}(s) e^{-\int_{a}^{s}\left(\lambda_{\varepsilon}+\varepsilon v(\xi)\right) \mathrm{d} \xi} \mathrm{d} s
\end{aligned}
$$

with $\bar{\pi}_{1}$ and $\pi_{2}$ are defined in (3.5).

In view of (5.5) the function $h_{\epsilon}$ is positive and by a simple computation we get,

$$
\begin{aligned}
h_{\varepsilon}(a) & =\int_{a}^{\infty}[(\bar{N}-\varepsilon) \beta(s) \\
& \left.+\frac{\delta}{\mu+\delta+\varepsilon} v(s)(\psi(\bar{N})-\varepsilon) \int_{s}^{\infty} \phi(\sigma) \frac{\pi_{2}(\sigma)}{\pi_{2}(s)} e^{-\varepsilon(\sigma-s)} \mathrm{d} \sigma\right] \frac{\bar{\pi}_{1}(s)}{\bar{\pi}_{1}(a)} e^{-\int_{a}^{s}\left(\lambda_{\varepsilon}+\varepsilon v(\xi)\right) \mathrm{d} \xi} \mathrm{d} s \\
& =\int_{a}^{\infty}\left[(\bar{N}-\varepsilon) \beta(s)+v(s)(\psi(\bar{N})-\varepsilon) k_{\epsilon}(s)\right] \frac{\bar{\pi}_{1}(s)}{\bar{\pi}_{1}(a)} e^{-\int_{a}^{s}\left(\lambda_{\varepsilon}+\varepsilon v(\xi)\right) \mathrm{d} \xi} \mathrm{d} s .
\end{aligned}
$$


These two equations satisfy

$$
k_{\varepsilon}^{\prime}(a)-(\mu+\phi(a)+\varepsilon) k_{\varepsilon}(a)=-\frac{\delta}{\mu+\delta+\varepsilon} \phi(a),
$$

and

$$
h_{\varepsilon}^{\prime}(a)-\left(\lambda_{\varepsilon}+\mu+v(a)(\psi(\bar{N})+\varepsilon)\right) h_{\varepsilon}(a)=-(\bar{N}-\varepsilon) \beta(a)-v(a)(\psi(\bar{N})-\varepsilon) k_{\varepsilon}(a) .
$$

Now by simple computations we have

$$
\begin{aligned}
\frac{\mathrm{d}}{\mathrm{d} t} \int_{0}^{\infty} h_{\varepsilon}(a) \tilde{i}(t, a) \mathrm{d} a & =-\int_{0}^{\infty} h_{\varepsilon}(a) \frac{\partial}{\partial a} \tilde{i}(t, a) \mathrm{d} a-\int_{0}^{\infty} h_{\varepsilon}(a)(\mu+v(a)(\psi(\bar{N})+\varepsilon)) \tilde{i}(t, a) \mathrm{d} a \\
& =h_{\epsilon}(0) \tilde{i}(t, 0)+\int_{0}^{\infty} h_{\varepsilon}^{\prime}(a) \tilde{i}(t, a) \mathrm{d} a \\
& -\int_{0}^{\infty} h_{\varepsilon}(a)(\mu+v(a)(\psi(\bar{N})+\varepsilon)) \tilde{i}(t, a) \mathrm{d} a
\end{aligned}
$$

Using (5.7), $h_{\epsilon}(0)=1$ and the expression of $\tilde{i}(t, 0)$ we obtain

$$
\frac{\mathrm{d}}{\mathrm{d} t} \int_{0}^{\infty} h_{\varepsilon}(a) \tilde{i}(t, a) \mathrm{d} a=\lambda_{\epsilon} \int_{0}^{\infty} h_{\varepsilon}(a) \tilde{i}(t, a) \mathrm{d} a-\int_{0}^{\infty} v(a)(\psi(\bar{N})-\varepsilon) k_{\varepsilon}(a) \tilde{i}(t, a) \mathrm{d} a+\delta \tilde{R} .
$$

Now, from (5.6) we have

$$
\begin{aligned}
\frac{\mathrm{d}}{\mathrm{d} t} \int_{0}^{\infty} k_{\varepsilon}(a) \tilde{q}(t, a) \mathrm{d} a & =\int_{0}^{\infty}\left(k_{\varepsilon}^{\prime}(a)-(\mu+\phi(a)) k_{\varepsilon}(a)\right) \tilde{q}(t, a) \mathrm{d} a \\
& +\int_{0}^{\infty} v(a)(\psi(\bar{N})-\varepsilon) k_{\varepsilon}(a) \tilde{i}(t, a) \mathrm{d} a \\
& =\epsilon \int_{0}^{\infty} k_{\epsilon}(a) \tilde{q}(t, a) \mathrm{d} a-\frac{\delta}{\mu+\delta+\epsilon} \int_{0}^{\infty} \phi(a) \tilde{q}(t, a) \mathrm{d} a \\
& +\int_{0}^{\infty} v(a)(\psi(\bar{N})-\varepsilon) k_{\varepsilon}(a) \tilde{i}(t, a) \mathrm{d} a
\end{aligned}
$$

Set $I_{\varepsilon}(t)=\int_{0}^{\infty} h_{\varepsilon}(a) \tilde{i}(t, a) \mathrm{d} a+\int_{0}^{\infty} k_{\varepsilon}(a) \tilde{q}(t, a) \mathrm{d} a+\frac{\delta}{\mu+\delta+\varepsilon} \tilde{R}(t)$, and using these two last equalities we get

$$
\begin{aligned}
I_{\varepsilon}^{\prime}(t) & =\lambda_{\varepsilon} \int_{0}^{\infty} h_{\varepsilon}(a) \tilde{i}(t, a) \mathrm{d} a+\varepsilon \int_{0}^{\infty} k_{\varepsilon}(a) \tilde{q}(t, a) \mathrm{d} a+\varepsilon \frac{\delta}{\mu+\delta+\varepsilon} \tilde{R}(t), \\
& \geq \max \left\{\lambda_{\varepsilon}, \varepsilon\right\} I_{\varepsilon}(t) .
\end{aligned}
$$

This implies that $I_{\varepsilon}(t) \geq e^{\max \left\{\lambda_{\varepsilon}, \varepsilon\right\} t} I_{\varepsilon}(0)$. Consequently, the boundedness of $h_{\varepsilon}$ and $k_{\varepsilon}$ lead to $\limsup _{t \rightarrow \infty} B(t)=\infty$ in $\Omega_{0}$, which is a contradiction. The lemma is proved.

From Theorem 5.7 in [28] we have 
Proposition 5.5. There exists a compact attractor $\mathcal{A}_{1}$ that attracts all solutions with initial data $\phi_{0}$ in $\Omega_{0}$. Moreover $\mathcal{A}_{1}$ is $\rho$ - uniformly persistent, that is, there exists $C>0$ such that

$$
B(t):=i(t, 0) \geq C \text { for all } \phi_{0} \in \mathcal{A}_{1} \text {. }
$$

We give now some estimates of solutions of (3.4).

Lemma 5.6. For all $\phi_{0} \in \mathcal{A}_{1}$ The following estimates are satisfied

$$
\frac{i(t, a)}{i^{*}(a)} \geq \frac{C}{i^{*}(0)}, \quad \frac{J(t)}{J^{*}} \geq \frac{C}{i^{*}(0)}, \frac{R(t)}{R^{*}} \geq \frac{C}{i^{*}(0)},
$$

for all $t \in \mathbb{R}$ and $a \geq 0$ and $C$ is defined in Proposition 5.5.

Proof. By (3.4), (5.1) and Proposition 5.5 we easily have

$$
\frac{i(t, a)}{i^{*}(a)}=\frac{B(t-a)}{i^{*}(0)} \geq \frac{C}{i^{*}(0)},
$$

and

$$
\frac{J(t)}{J^{*}} \geq \frac{C}{i^{*}(0)},
$$

for all $t \in \mathbb{R}$ and $a \geq 0$. Next, again from (3.4), (5.1) and Proposition 5.5,

$$
\begin{aligned}
q(t, a)=B(t-a) F(a) & \geq C F(a) \\
& \geq \frac{C}{i^{*}(0)} q^{*}(a),
\end{aligned}
$$

with $F(a)=\pi_{2}(a) \int_{0}^{a} v(\sigma) \psi(\bar{N}) \frac{\bar{\pi}_{1}(\sigma)}{\pi_{2}(\sigma)} \mathrm{d} \sigma$.

This, with the equation of $R$ in (3.4), gives

$$
R^{\prime}(t) \geq \frac{C}{i^{*}(0)} \int_{0}^{\infty} \phi(a) q^{*}(a) \mathrm{d} a-(\mu+\delta) R(t) .
$$

Using the expression of $R^{*}$ stated in (5.1), we get

$$
R^{\prime}(t) \geq \frac{C}{i^{*}(0)}(\mu+\delta) R^{*}-(\mu+\delta) R(t)
$$

Let $r \in \mathbb{R}$ and $t \geq r$, then integrating this last inequation over $(r, t)$ we find

$$
R(t) \geq\left(R(r)-\frac{C R^{*}}{i^{*}(0)}\right) e^{(\mu+\delta)(r-t)}+\frac{C R^{*}}{i^{*}(0)},
$$

passing to the limit as $r \rightarrow-\infty$ we conclude that

$$
\frac{R(t)}{R^{*}} \geq \frac{C}{i^{*}(0)},
$$


for all $t \in \mathbb{R}$. The lemma is proved.

Now we turn to the global stability of the positive equilibrium.

Theorem 5.7. Suppose that $R_{0}>1$. Then the positive equilibrium is globally asymptotically stable in $\Omega_{0}$.

Proof. We set

$$
k(a)=\frac{i^{*}(0)}{S^{*}}-i^{*}(0) \int_{0}^{a} \beta(\sigma) \bar{\pi}_{1}(\sigma) \mathrm{d} \sigma-\frac{\delta R^{*}}{S^{*}} \int_{0}^{a} \frac{\phi(\sigma) F(\sigma)}{\int_{0}^{\infty} \phi(\xi) F(\xi) \mathrm{d} \xi} \mathrm{d} \sigma
$$

with $F(a)=\pi_{2}(a) \int_{0}^{a} v(\sigma) \psi(\bar{N}) \frac{\bar{\pi}_{1}(\sigma)}{\pi_{2}(\sigma)} \mathrm{d} \sigma$.

\section{Remark 5.8.}

1) $k(a) \geq 0$ for all $a \geq 0$. Indeed, from the last equation of (5.1), the function $k$ may be rewritten as

$$
\begin{aligned}
k(a) & =J^{*}-i^{*}(0) \int_{0}^{a} \beta(\sigma) \bar{\pi}_{1}(\sigma) \mathrm{d} \sigma+\frac{\delta R^{*}}{S^{*}}\left(1-\int_{0}^{a} \frac{\phi(\sigma) F(\sigma)}{\int_{0}^{\infty} \phi(\xi) F(\xi) \mathrm{d} \xi} \mathrm{d} \sigma\right), \\
& =i^{*}(0) \int_{a}^{\infty} \beta(\sigma) \bar{\pi}_{1}(\sigma) \mathrm{d} \sigma+\frac{\delta R^{*}}{S^{*}} \int_{a}^{\infty} \frac{\phi(\sigma) F(\sigma)}{\int_{0}^{\infty} \phi(\xi) F(\xi) \mathrm{d} \xi} \mathrm{d} \sigma .
\end{aligned}
$$

2) $k, k^{\prime} \in L^{1}\left(\mathbf{R}^{+}\right)$. In fact, from Fubini theorem we find

$$
\int_{0}^{\infty} k(a) \mathrm{d} a=i^{*}(0) \int_{0}^{\infty} \sigma \beta(\sigma) \bar{\pi}_{1}(\sigma) \mathrm{d} \sigma+\frac{\delta R^{*}}{S^{*}} \int_{0}^{\infty} \frac{\sigma \phi(\sigma) F(\sigma)}{\int_{0}^{\infty} \phi(\xi) F(\xi) \mathrm{d} \xi} \mathrm{d} \sigma .
$$

Observe that these last integrals are finite. Finally, $k^{\prime} \in L^{1}$ can be easily showed.

Let $\phi_{0} \in \mathcal{A}_{1}$, since $\mathcal{A}_{1}$ is invariant, then there exists a total trajectory $\Psi_{1}: \mathbb{R} \rightarrow \mathcal{A}_{1}$, such that $\Psi_{1}(t)=$ $(S(t), i(t,),. q(t,),. R(t))$ is solution of (3.4) passing through $\phi_{0}$. Let $H(x)=x-\ln (x)-1$, and introduce the following Lyapunov functional

$$
V\left(\Psi_{1}(t)\right)=H\left(\frac{S(t)}{S^{*}}\right)+\int_{0}^{\infty} k(a) H\left(\frac{i(t, a)}{i^{*}(a)}\right) \mathrm{d} a+\frac{\delta R^{*}}{S^{*}(\mu+\delta)} H\left(\frac{R(t)}{R^{*}}\right)
$$

We differentiate the first term of $V$ we have

$$
\frac{\mathrm{d}}{\mathrm{d} t} H\left(\frac{S(t)}{S^{*}}\right)=\frac{1}{S^{*}}\left(1-\frac{S^{*}}{S(t)}\right)(A-\mu S(t)-S(t) J(t))
$$

using the fact that $A=\mu S^{*}+S^{*} J^{*}$, we obtain

$$
I_{1}^{\prime}(t):=\frac{\mathrm{d}}{\mathrm{d} t} H\left(\frac{S(t)}{S^{*}}\right)=\mu\left(1-\frac{S^{*}}{S(t)}\right)\left(1-\frac{S(t)}{S^{*}}\right)-\frac{S(t)}{S^{*}} J(t)+J(t)+\left(1-\frac{S^{*}}{S(t)}\right) J^{*} .
$$


Concerning the second term of $V$, using the second equation of (3.4) and following the same arguments as in the proof of Lemma 9.18 in [28] we get,

$$
\begin{aligned}
I_{2}^{\prime}(t)=\frac{\mathrm{d}}{\mathrm{d} t} \int_{0}^{\infty} k(a) H\left(\frac{i(t, a)}{i^{*}(a)}\right) \mathrm{d} a & =k(0) H\left(\frac{i(t, 0)}{i^{*}(0)}\right)+\int_{0}^{\infty} k^{\prime}(a) H\left(\frac{i(t, a)}{i^{*}(a)}\right) \mathrm{d} a \\
& =\frac{i^{*}(0)}{S^{*}} H\left(\frac{S(t) J(t)+\delta R(t)}{i^{*}(0)}\right)+\int_{0}^{\infty} k^{\prime}(a) H\left(\frac{B(t-a)}{i^{*}(0)}\right) \mathrm{d} a
\end{aligned}
$$

with $B(t)=i(t, 0)$.

From the last equation of (5.1), we have $\frac{S^{*} J^{*}}{i^{*}(0)}+\frac{\delta R^{*}}{i^{*}(0)}=1$. Combining this, with the fact that $H$ is convex, we find

$$
\begin{aligned}
H\left(\frac{S(t) J(t)+\delta R(t)}{i^{*}(0)}\right) & =H\left(\frac{S^{*} J^{*}}{i^{*}(0)} \frac{S(t) J(t)}{S^{*} J^{*}}+\frac{\delta R^{*}}{i^{*}(0)} \frac{R(t)}{R^{*}}\right) \\
& \leq \frac{S^{*} J^{*}}{i^{*}(0)} H\left(\frac{S(t) J(t)}{S^{*} J^{*}}\right)+\frac{\delta R^{*}}{i^{*}(0)} H\left(\frac{R(t)}{R^{*}}\right), \\
& \leq \frac{S^{*} J^{*}}{i^{*}(0)}\left(\frac{S(t) J(t)}{S^{*} J^{*}}-\ln \left(\frac{S(t)}{S^{*}}\right)-\ln \left(\frac{J(t)}{J^{*}}\right)-1\right)+\frac{\delta R^{*}}{i^{*}(0)} H\left(\frac{R(t)}{R^{*}}\right) .
\end{aligned}
$$

Using this last inequality in (5.10),

$$
\begin{aligned}
I_{2}^{\prime}(t) & \leq J^{*}\left(\frac{S(t) J(t)}{S^{*} J^{*}}-\ln \left(\frac{S(t)}{S^{*}}\right)-\ln \left(\frac{J(t)}{J^{*}}\right)-1\right)+\frac{\delta R^{*}}{S^{*}} H\left(\frac{R(t)}{R^{*}}\right) \\
& +\int_{0}^{\infty} k^{\prime}(a) H\left(\frac{B(t-a)}{i^{*}(0)}\right) \mathrm{d} a .
\end{aligned}
$$

Summing $I_{1}^{\prime}$ and $I_{2}^{\prime}$,

$$
\begin{aligned}
I_{1}^{\prime}(t)+I_{2}^{\prime}(t) & \leq \mu\left(1-\frac{S^{*}}{S(t)}\right)\left(1-\frac{S(t)}{S^{*}}\right)-J^{*} H\left(\frac{S^{*}}{S(t)}\right)+J^{*} H\left(\frac{J(t)}{J^{*}}\right)+\frac{\delta R^{*}}{S^{*}} H\left(\frac{R(t)}{R^{*}}\right) \\
& +\int_{0}^{\infty} k^{\prime}(a) H\left(\frac{B(t-a)}{i^{*}(0)}\right) \mathrm{d} a .
\end{aligned}
$$


Using Jensen inequality, we have

$$
\begin{aligned}
J^{*} H\left(\frac{J(t)}{J^{*}}\right) & =J^{*} H\left(\frac{\int_{0}^{\infty} \beta(a) \bar{\pi}_{1}(a) B(t-a) \mathrm{d} a}{i^{*}(0) \int_{0}^{\infty} \beta(\xi) \bar{\pi}_{1}(\xi) \mathrm{d} \xi}\right) \\
& \leq \frac{J^{*}}{\int_{0}^{\infty} \beta(\xi) \pi_{1}(\xi) \mathrm{d} \xi} \int_{0}^{\infty} H\left(\frac{B(t-a)}{i^{*}(0)}\right) \beta(a) \bar{\pi}_{1}(a) \mathrm{d} a \\
& \leq i^{*}(0) \int_{0}^{\infty} H\left(\frac{B(t-a)}{i^{*}(0)}\right) \beta(a) \bar{\pi}_{1}(a) \mathrm{d} a .
\end{aligned}
$$

Therefore

$$
\begin{aligned}
I_{1}^{\prime}(t)+I_{2}^{\prime}(t) & \leq \mu\left(1-\frac{S^{*}}{S(t)}\right)\left(1-\frac{S(t)}{S^{*}}\right)-J^{*} H\left(\frac{S^{*}}{S(t)}\right)+\frac{\delta R^{*}}{S^{*}} H\left(\frac{R(t)}{R^{*}}\right) \\
& +\int_{0}^{\infty}\left(k^{\prime}(a)+\beta(a) \bar{\pi}_{1}(a) i^{*}(0)\right) H\left(\frac{B(t-a)}{i^{*}(0)}\right) \mathrm{d} a
\end{aligned}
$$

Since

$$
k^{\prime}(a)=-i^{*}(0) \beta(a) \bar{\pi}_{1}(a)-\frac{\delta R^{*}}{S^{*}} \frac{\phi(a) F(a)}{\int_{0}^{\infty} \phi(\xi) F(\xi) \mathrm{d} \xi},
$$

then

$$
\begin{aligned}
I_{1}^{\prime}(t)+I_{2}^{\prime}(t) & \leq \mu\left(1-\frac{S^{*}}{S(t)}\right)\left(1-\frac{S(t)}{S^{*}}\right)-J^{*} H\left(\frac{S^{*}}{S(t)}\right)+\frac{\delta R^{*}}{S^{*}} H\left(\frac{R(t)}{R^{*}}\right) \\
& -\frac{\delta R^{*}}{S^{*}} \int_{0}^{\infty} H\left(\frac{B(t-a)}{i^{*}(0)}\right) \frac{\phi(a) F(a)}{\int_{0}^{\infty} \phi(\xi) F(\xi) \mathrm{d} \xi} \mathrm{d} a .
\end{aligned}
$$

Now we focus on the third term of $V$

$$
\begin{aligned}
I_{3}^{\prime}(t) & :=\frac{\delta R^{*}}{S^{*}(\mu+\delta)} \frac{\mathrm{d}}{\mathrm{d} t} H\left(\frac{R(t)}{R^{*}}\right) \\
& =\frac{\delta R^{*}}{S^{*}(\mu+\delta)} H^{\prime}\left(\frac{R(t)}{R^{*}}\right)\left(\frac{i^{*}(0)}{R^{*}} \int_{0}^{\infty} \phi(a) F(a) \frac{B(t-a)}{i^{*}(0)} \mathrm{d} a-(\mu+\delta) \frac{R(t)}{R^{*}}\right) .
\end{aligned}
$$

Since

$$
R^{*}=\frac{i^{*}(0)}{\mu+\delta} \int_{0}^{\infty} \phi(a) F(a) \mathrm{d} a
$$


then

$$
I_{3}^{\prime}(t)=\frac{\delta R^{*}}{S^{*}} H^{\prime}\left(\frac{R(t)}{R^{*}}\right) \int_{0}^{\infty} \frac{\phi(a) F(a)}{\int_{0}^{\infty} \phi(\xi) F(\xi) \mathrm{d} \xi} \frac{B(t-a)}{i^{*}(0)} \mathrm{d} a-\frac{\delta R(t)}{S^{*}} H^{\prime}\left(\frac{R(t)}{R^{*}}\right)
$$

For $I(t):=V\left(\Psi_{1}(t)\right)=I_{1}(t)+I_{2}(t)+I_{3}(t)$, we have

$$
\begin{aligned}
I^{\prime}(t) & \leq \mu\left(1-\frac{S^{*}}{S(t)}\right)\left(1-\frac{S(t)}{S^{*}}\right)-J^{*} H\left(\frac{S^{*}}{S(t)}\right)+\frac{\delta R^{*}}{S^{*}} H\left(\frac{R(t)}{R^{*}}\right) \\
& -\frac{\delta R^{*}}{S^{*}} \int_{0}^{\infty} H\left(\frac{B(t-a)}{i^{*}(0)}\right) \frac{\phi(a) F(a)}{\int_{0}^{\infty} \phi(\xi) F(\xi) \mathrm{d} \xi} \mathrm{d} a \\
& +\frac{\delta R^{*}}{S^{*}} H^{\prime}\left(\frac{R(t)}{R^{*}}\right) \int_{0}^{\infty} \frac{\phi(a) F(a)}{\int_{0}^{\infty} \phi(\xi) F(\xi) \mathrm{d} \xi} \frac{B(t-a)}{i^{*}(0)} \mathrm{d} a-\frac{\delta R(t)}{S^{*}} H^{\prime}\left(\frac{R(t)}{R^{*}}\right) .
\end{aligned}
$$

Finally,

$$
\begin{aligned}
I^{\prime}(t) & \leq \mu\left(1-\frac{S^{*}}{S(t)}\right)\left(1-\frac{S(t)}{S^{*}}\right)-J^{*} H\left(\frac{S^{*}}{S(t)}\right) \\
& +\frac{\delta R^{*}}{S^{*}} \int_{0}^{\infty}\left[H\left(\frac{R(t)}{R^{*}}\right)-H\left(\frac{B(t-a)}{i^{*}(0)}\right)+H^{\prime}\left(\frac{R(t)}{R^{*}}\right)\left(\frac{B(t-a)}{i^{*}(0)}-\frac{R(t)}{R^{*}}\right)\right] \frac{\phi(a) F(a)}{\int_{0}^{\infty} \phi(\xi) F(\xi) \mathrm{d} \xi} \mathrm{d} .
\end{aligned}
$$

Since $H(x)-H(y)+H^{\prime}(x)(y-x) \leq 0$ then the third term of (5.11) is negative and therefore $I \leq 0$.

Further, notice that $\frac{\mathrm{d}}{\mathrm{d} t} V\left(\Psi_{1}(t)\right)=0$ implies that $S(t)=S^{*}$ and $\frac{B(t-a)}{i^{*}(0)}=\frac{R(t)}{R^{*}}$ for all $t \in \mathbb{R}$ and $a \geq 0$. From the equation of $S$ in (3.4) we have $J(t)=J^{*}$ for all $t \in \mathbb{R}$. This leads to

$$
\begin{aligned}
J^{*} & =\int_{0}^{\infty} \beta(a) \bar{\pi}_{1}(a) B(t-a) \mathrm{d} a, \\
& =\frac{R(t)}{R^{*}} i^{*}(0) \int_{0}^{\infty} \beta(a) \bar{\pi}_{1}(a) \mathrm{d} a, \\
& =J^{*} \frac{R(t)}{R^{*}}
\end{aligned}
$$

and thus $R(t)=R^{*}$ for all $t \in \mathbb{R}$. It yields $B(t-a)=i^{*}(0)$ for all $t \in \mathbb{R}$ and $a \geq 0$. Consequently $i(t, a)=i^{*}(a)$ and $q(t, a)=q^{*}(a)$ for all $t \in \mathbb{R}$ and $a \geq 0$. Finally, employing the same arguments as in the end of the proof of Theorem 4.1, we prove the global asymptotic stability of the endemic equilibrium. 


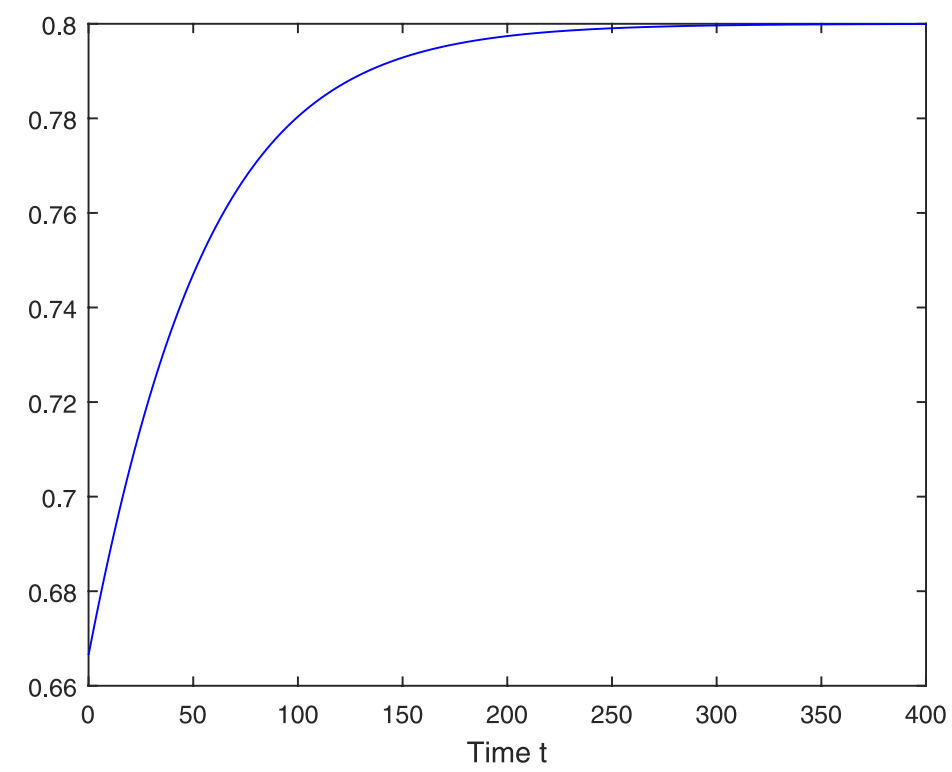

FIGURE 1. The evolution of function $\psi(N(t))$ with respect to time $t$.

\section{NUMERICAL SIMULATION}

The results of the previous sections are illustrated by numerical simulations. We consider the following values of parameters

$$
A=0.01, \quad \mu=0.02 \quad v(a) \equiv 0.1 \quad \text { and } \quad \psi(z)=\frac{1}{1+0.5 z},
$$

with the initial conditions

$$
S_{0}=0.9, \quad i_{0}(a)=10^{-2} e^{-0.1 a}, \quad q_{0}(a)=0, \quad \text { and } \quad r_{0}=0
$$

The functions $\beta$ and $\phi$ are chosen as

$$
\beta(a)= \begin{cases}0, & \text { if } a \leq 5, \\ 8 \times 10^{-3}(a-5)^{2} e^{-0.1(a-5)}, & \text { if } a>5,\end{cases}
$$

and

$$
\phi(a)= \begin{cases}0, & \text { if } a \leq 10 \\ 8 \times 10^{-3}(a-10)^{2} e^{(-0.1(a-10))} ; & \text { if } a>10 .\end{cases}
$$

In the first case, we fix $\delta=0$ we then find $R_{0}<1$. According to Theorem 4.1, the disease-free equilibrium is globally asymptotically stable see Figures 2 and 3 .

In the second case, we take $\delta=0.4$ and then $R_{0}>1$. From Theorem 5.7, the positive equilibrium is globally asymptotically stable see Figures 4 and 5 . 

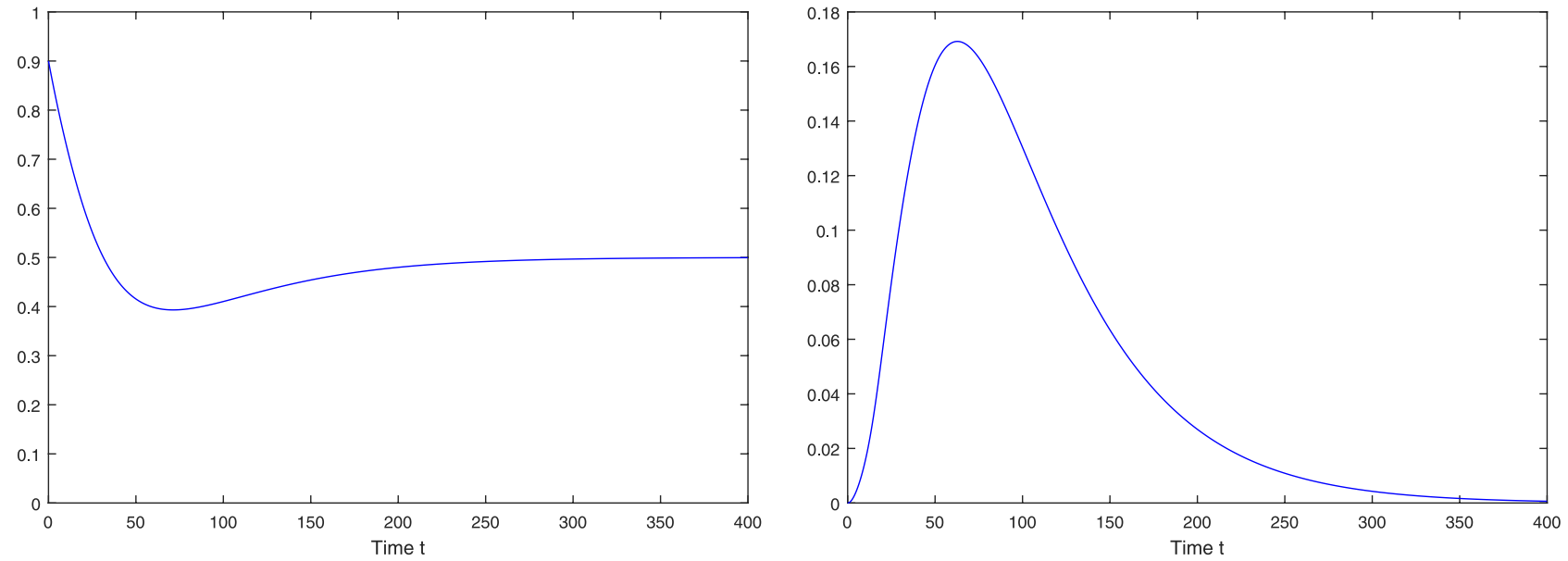

FiguRE 2. The evolution of solution $S$ (left) and $R$ (right) with respect to time $t$.
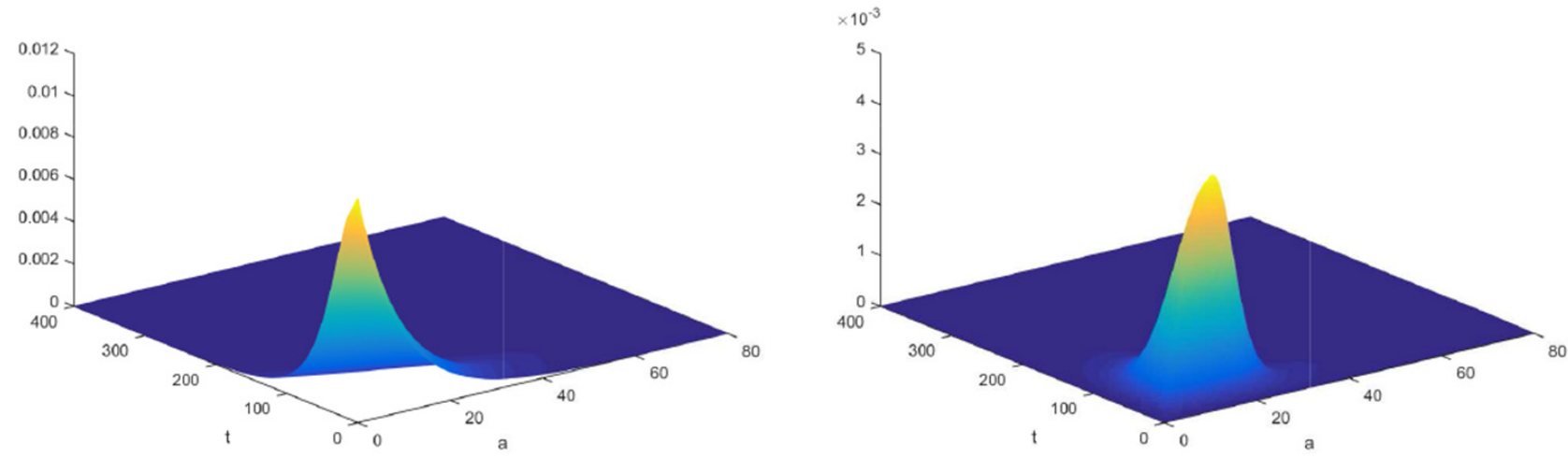

FIGURE 3. The evolution of solutions $i$ (left) and $q$ (right) with respect to time $t$ and age $a$.

Now, for the following parameters, $v(a) \equiv 0.2$ and

$$
\phi(a)= \begin{cases}0, & \text { if } a \leq 10 \\ 4 \times 10^{-3}(a-10)^{2} e^{(-0.1(a-10))} ; & \text { if } a>10\end{cases}
$$

we obtain $R_{0}<1$ for $\delta \in\{0,0.1,0.5\}$.

Next we choose the following parameters $A=0.015, \mu=0.01$ and $v(a) \equiv 0.1$,

$$
\beta(a)= \begin{cases}0, & \text { if } a \leq 5, \\ 9 \times 10^{-3}(a-5)^{2} e^{-0.1(a-5)}, & \text { if } a>5,\end{cases}
$$



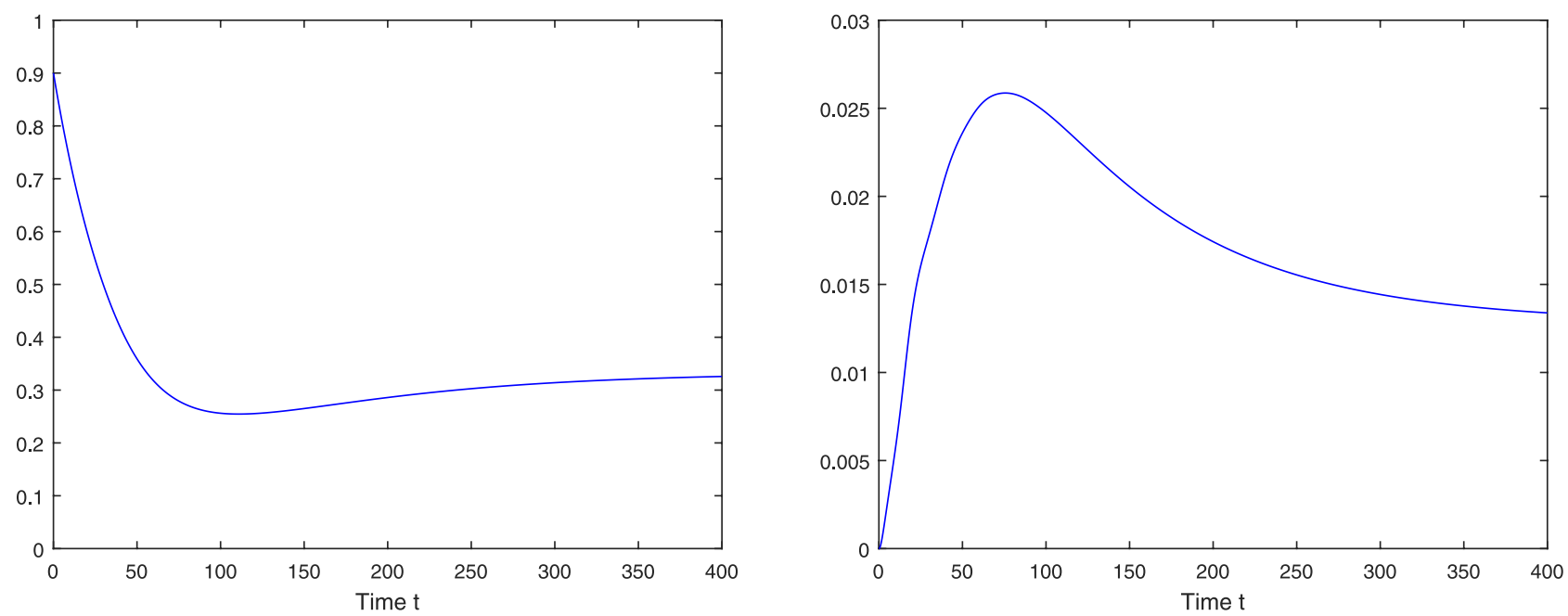

FIGURE 4. The evolution of solution $S$ (left) and $R$ (right) with respect to time $t$.
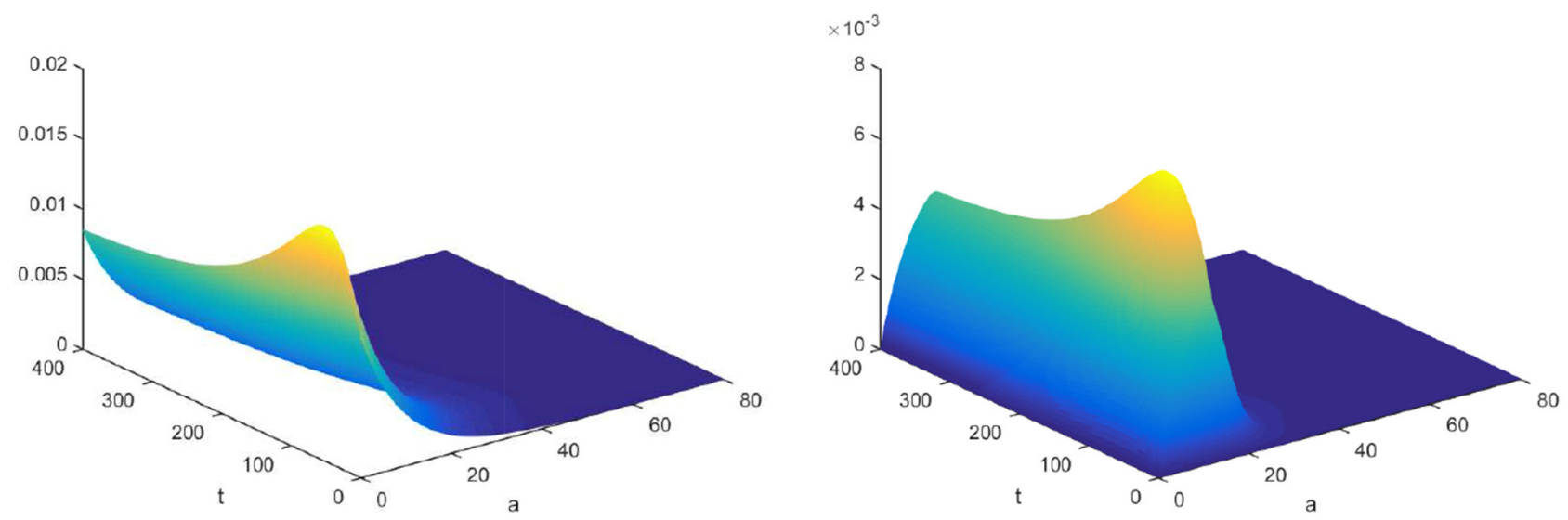

FigURE 5. The evolution of solutions $i$ (left) and $q$ (right) with respect to time $t$ and age $a$.

and

$$
\phi(a)= \begin{cases}0, & \text { if } a \leq 10 \\ 8 \times 10^{-3}(a-10)^{2} e^{-0.1(a-10)}, & \text { if } a>10 .\end{cases}
$$

So, we find $R_{0}>1$ for all $\delta \geq 0$.

\subsection{Discussion}

In this paper we presented an age controlled structured epidemic model of Susceptible-Infected-QuarantineRecovered-Infected (SIQRI) type where we will look for the role of individuals that leave the $R$-class before 


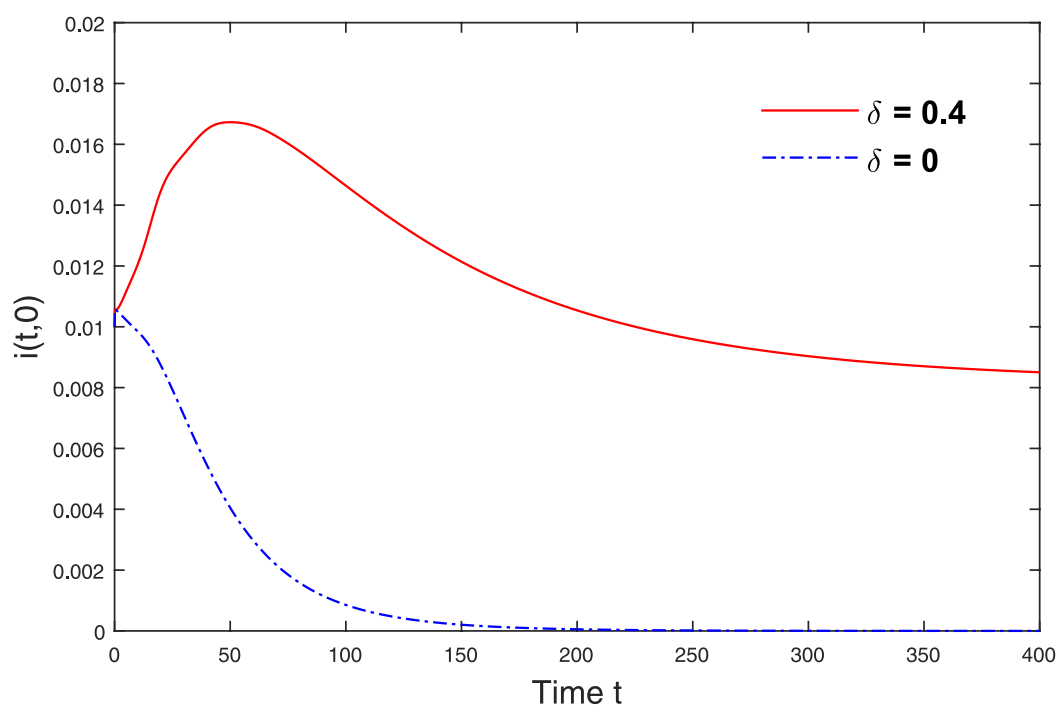

FiguRE 6 . The impact of the relapse parameter $\delta$ on the incidence $i(t, 0)$. For $\delta=0, R_{0}$ is less than one. For $\delta=0.4, R_{0}$ becomes greater than one.

being completely recovered and thus will participate again to the transmission of the disease. The epidemic reproduction number $R_{0}$ is a sum of two epidemic reproduction numbers. The first one $R_{00}=\int_{0}^{\infty} \bar{N} \beta(s) \bar{\pi}_{1}(s) \mathrm{d} s$ corresponds to the basic epidemic number in the case of an SIQR system. It means that there is no relapse of the individuals in the recovered class $\mathrm{R}$. The second one $R_{o r}=\frac{\delta}{\delta+\mu} \psi(\bar{N}) \int_{0}^{\infty} \phi(\sigma) \pi_{2}(\sigma) \int_{0}^{\sigma} v(s) \frac{\bar{\pi}_{1}(s)}{\pi_{2}(s)} \mathrm{d} s \mathrm{~d} \sigma$ corresponds to the average number of people newly infected by a R-class person who has relapsed. It can be noted that $R_{0}$ increases with the relapse rate $\delta$.

Some new global stability results are obtained for this age structured SIQRI model including a screening strategy and a quarantine program; and to highlight the influence of these two public health measures, we presented a series of simulations based on each parameter separately.

By fixing all the parameters and in particular the screening rate at 0.1 and varying the relapse rate we obtain - In the case where there is no relapse and $R_{0}<1$. The incidence function decreases to 0 (see Fig. 6), this leads to the convergence of solutions to the disease-free equilibrium (DFE).

- When we increase the relapse rate to 0.4 we obtain $R_{0}>1$. The incidence function takes strictly positive values (see Fig. 6), in this case there is convergence of solutions to the endemic equilibrium (EE).

However, when we focus our efforts on the screening program by doubling the screening rate, we can get $R_{0}<1$ even when we have treatment and when the relapse rate is not zero $(\delta \in\{0,0.1,0.5\}) ;$ Figure 9 shows that the incidence rate becomes approximately identically zero at a final time $t_{f}=400$. In this case, the effort on the screening program gives $10 \%$ more individiuals added to the quarantine class.

Finally, by choosing other numerical values for the model parameters, we can get $R_{0}>1$ for every $\delta \geq 0$. In this case, Figure 12 shows that the incidence rate increases until $3 \%$ relatively to the total population. In fact, for $\delta=0$, we have $\frac{i\left(t_{f}, 0\right)}{N\left(t_{f}\right)}=0.73 \%$, while for $\delta=0.5$, we have $\frac{i\left(t_{f}, 0\right)}{N\left(t_{f}\right)}=3.2 \%$.

This means that when the relapse rate is sufficiently small, the incidence rate is less important and decreases until $0.73 \%$ suggesting a quarantine program. 

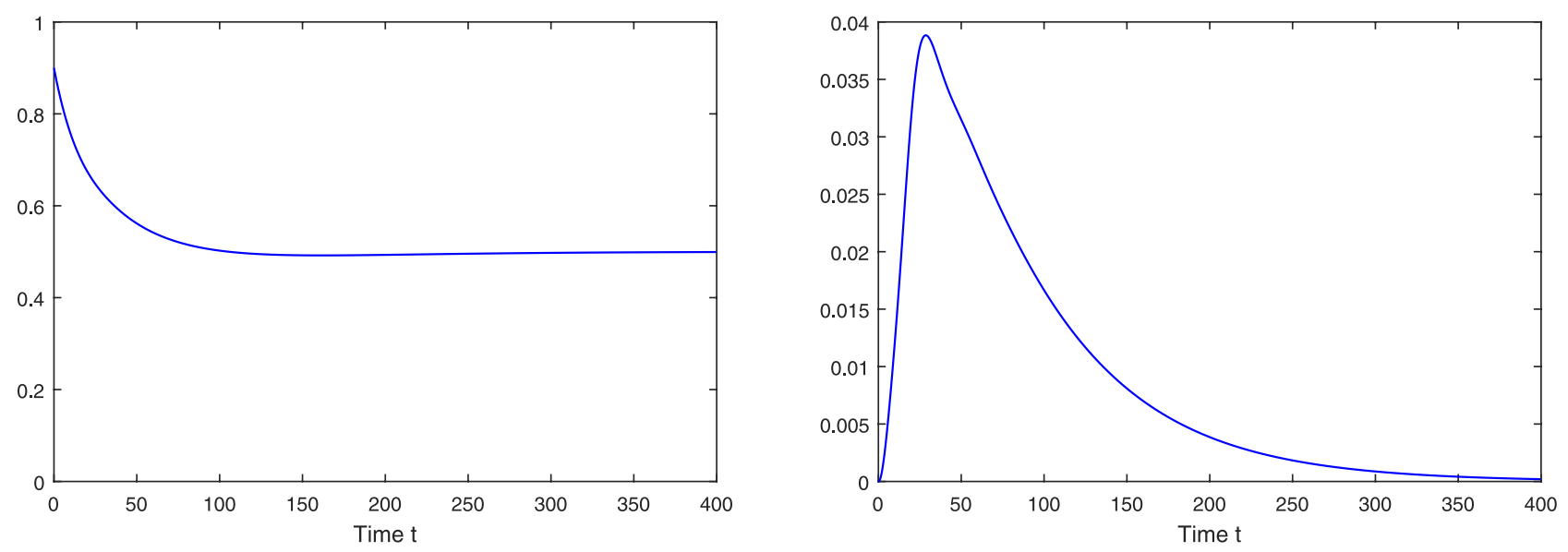

FIGURE 7. The evolution of solution $S$ (left) and $R$ (right) with respect to time $t$.
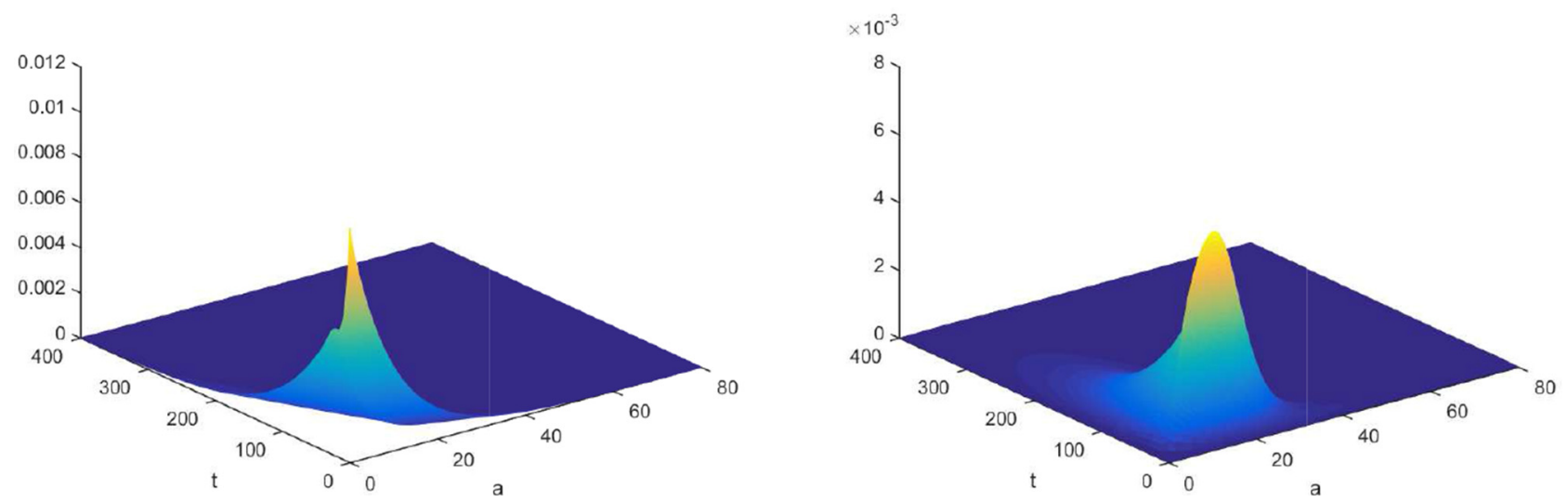

FIGURE 8 . The evolution of solutions $i$ (left) and $q$ (right) with respect to time $t$ and age $a$.

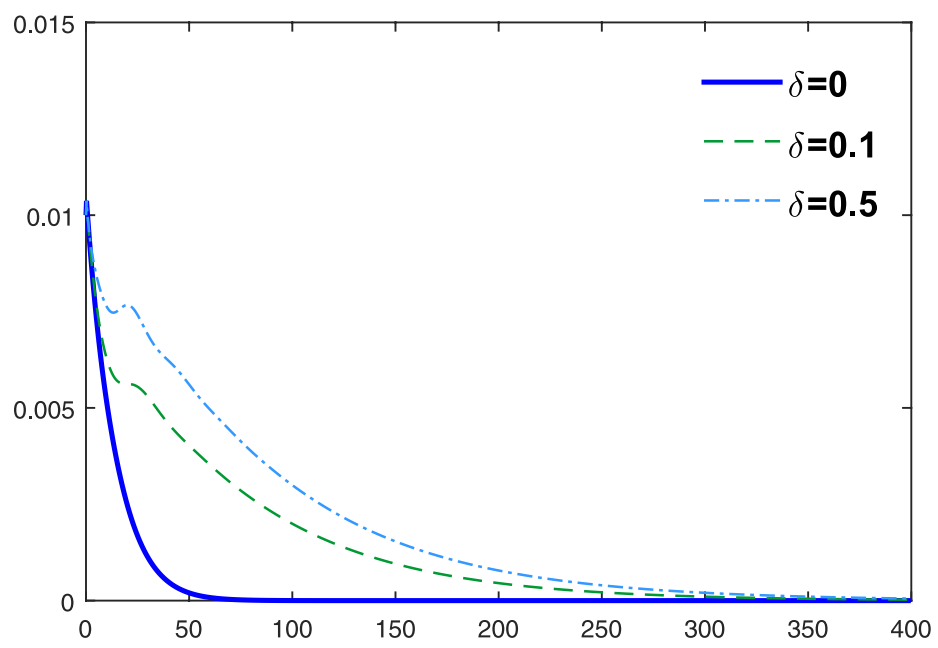

FIGURE 9. The evolution of $i(t, 0)$ with respect to time $t$. 

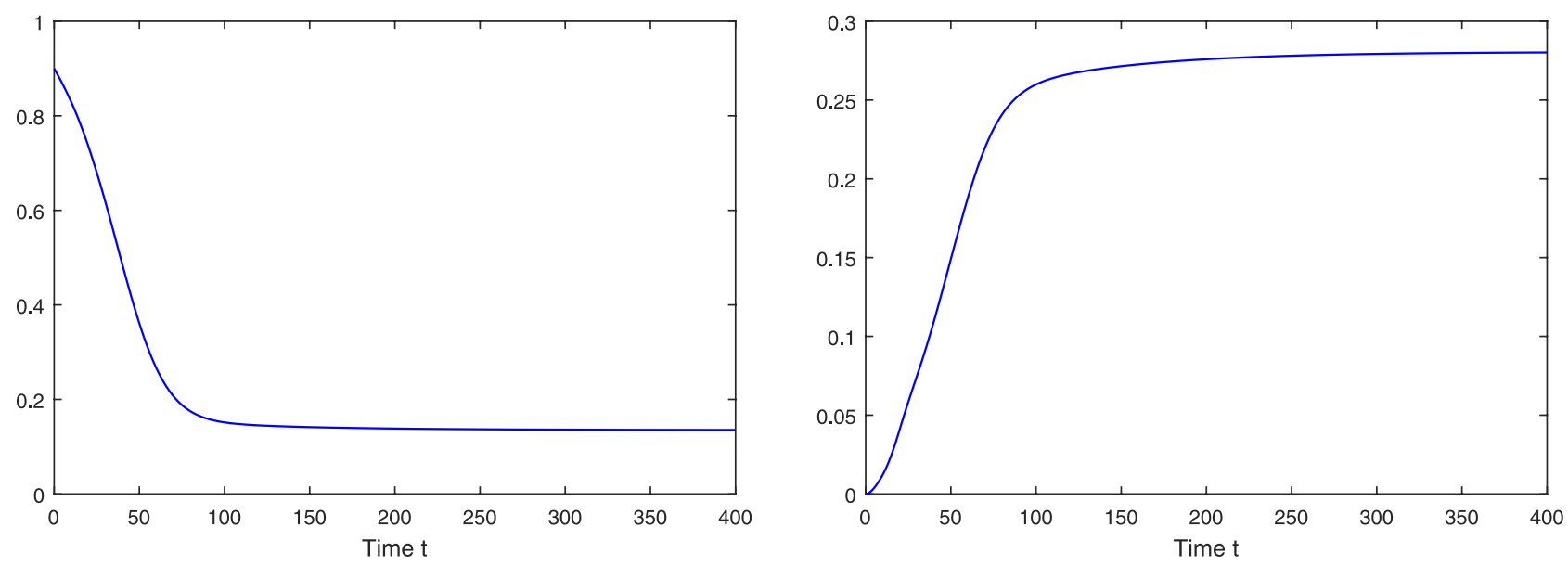

FiguRE 10. The evolution of solution $S$ (left) and $R$ (right) with respect to the time.
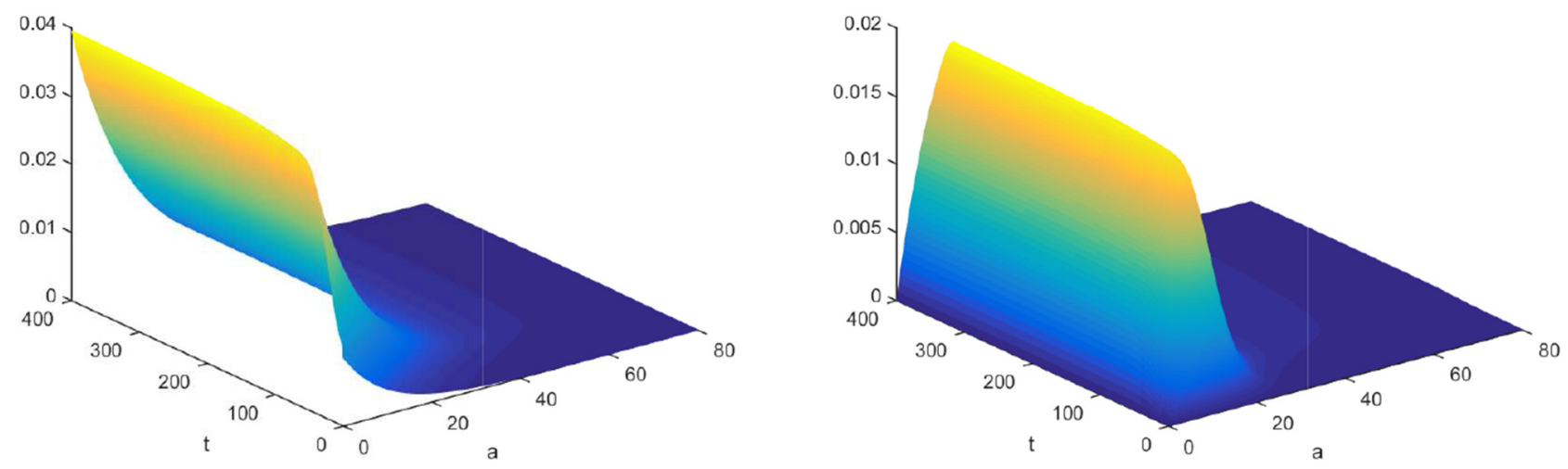

FIGURE 11. The evolution of solutions $i$ (left) and $q$ (right) with respect to time $t$ and age $a$.

These different simulations show the effect of the quarantine program on the incidence rate, and by the sequel on the asymptotic behaviour of the solutions. We can deduce that reducing transmissibility requires an efficient quarantine program. Moreover, a great effort in screening helps considerably in the detectability of the disease and in decreasing its spread.

As a perspective, it will be interesting to include in the proposed model, individuals leaving quarantine before it ends, to the best of our knowledge such a model has never been studied before, the quarantine interruption can generate many mathematical complications, especially in the construction of a suitable Lyapunov functional. 


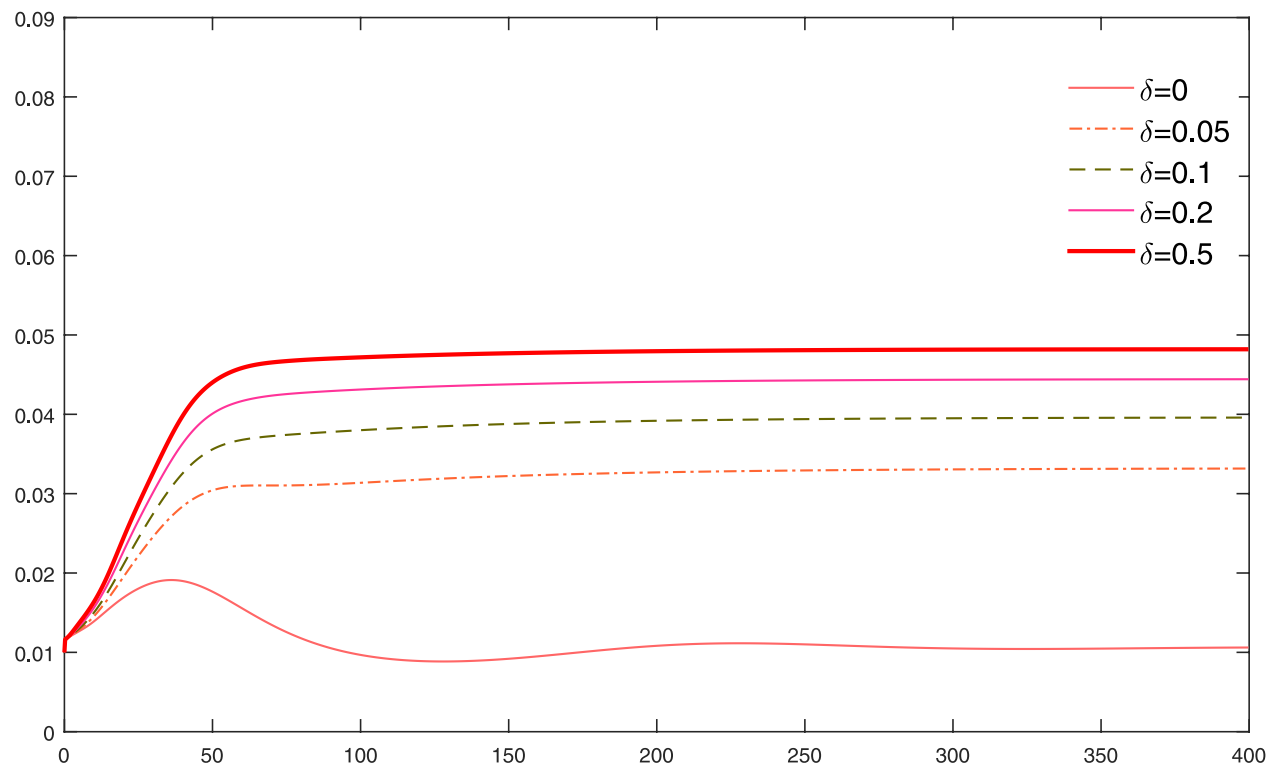

FIgURE 12. The evolution of the incidence $i(t, 0)$ with respect to time $t$ for different values of $\delta$.

Acknowledgements. The first and the second authors are partially supported by the DGRSDT, ALGERIA, project PRFU, code C00L03UN130120200004. The authors would like to thank the associate editor and the reviewers for their careful reading and helpful remarks.

\section{REFERENCES}

[1] B. Ainseba, Z. Feng, M. Iannelli and F.A. Milner, Control strategies for TB epidemics. Siam J. Appl. Math. 77 (2017) 82-107.

[2] B. Ainseba and M. Iannelli, Optimal screening in structured SIR epidemics. MMNP 7 (2012) 12-27.

[3] T.T. Ashezua, N.I. Akinwande, S. Abdulrahman, R.O. Dayiwola and F. Kuta, Local stability analysis of an infection age mathematical model for tuberculosis disease dynamics. J. Appl. Sci. Environ. Manag. 19 (2015) 665-669.

[4] S. Bentout, Y. Chen and S. Djilali, Global dynamics of an SEIR model with two age structures and a nonlinear incidence. Acta Appl. Math. 171 (2021) 1-27.

[5] S. Bentout and T.M. Touaoula, Global analysis of an infection age model with a class of nonlinear incidence rates. J. Math. Anal. Appl. 434 (2016) 1211-1239.

[6] D. Bernouilli, Essai d'une nouvelle analyse de la mortalité causée par la petite vérole et des avantages de l'inoculation pour la prévenir. Mém. Math. Phys. Acad. Roy. Sci., Paris 1760 (1766) 1-45.

[7] D. Bernouilli, Reflexions sur les avantages de l'inoculation. Mercure de France (1760) 173-190.

[8] I. Boudjema and T.M. Touaoula, Global stability of an infection and vaccination age-structured model with general nonlinear incidence. J. Nonlinear Funct. Anal. 33 (2018) 1-21.

[9] F. Brauer, Age infection in epidemiology models. Electr. J. Differ. Equ. Conf. 12 (2005) 29-37.

[10] C. Castillo-Chavez and Z. Feng, Mathematical models for the disease dynamics of the tuberculosis, Fourth International Conference on Mathematical Population Dynamics (1995).

[11] A. Chekroun, M.N. Frioui, T. Kuniya and T.M. Touaoula, Global stability of an age structured epidemic model with general Lyapunov functional. Math. Biosci. Eng. 16 (2019) 1525-1553.

[12] A. Chekroun, M.N. Frioui, T. Kuniya and T.M. Touaoula, Mathematical analysis of an age structured heroin-cocaine epidemic model. Discr. Continu. Dyn. Syst. B 25 (2020) 444-4477.

[13] Y. Chen, J. Yang and F. Zhang, The global stability for an SIRS model with infection age. Math. Biosci. Eng. 11 (2014) 449-469.

[14] O. Diekmann; J.A.P. Heesterbeek and J.AJ. Metz. On the definition and the computation of the basic reproduction ratio $R_{0}$ in models for infectious diseases in heterogeneous populations. J.Math. Biol. 28 (1990) 365-382.

[15] S. Djilali, T.M. Touaoula and S.E. Miri, A Heroin epidemic model: very general non linear incidence, treat-age, and global stability. Acta Appl. Math. Math. Appl. 52 (2017) 171-194. 
[16] M. Erdem, M. Safan and C. Castillo-Chavez, Mathematical Analysis of an SIQR influenza model with imperfect quarantine. Bull. Math. Biol. 79 (2017) doi: 10.1007/s11538-017-0301-6.

[17] M.N. Frioui, T.M. Touaoula and B. Ainseba, Global dynamics of an age structured model with relapse. Discrete Contin. Dyn. Syst. Ser. B (2020) doi: 10.3934/dcdsb.2019226.

[18] M. Iannelli, Mathematical Theory of Age- Structured Population Dynamics. Giardini Editori E Stampatori In Pisa (1994).

[19] M. Iannelli and F. Milner, The basic Approach to age structured population Dynamics: Models, Methods and Numerics. Lecture Notes on Mathematical Modelling in the Life Science (2017).

[20] W.O. Kermack and A.G. McKendrick, Contributions to the mathematical theory of epidemics 1. Proc. R. Soc. 115 (1927) $700-721$.

[21] T. Kuniya, Stability Analysis of an Age structured SIR model with a reduction method to EDOs. Mathematics 6 (2018) 147.

[22] J.P. LaSalle, The stability of dynamical systems. Regional conference series in applied mathematics, 25. SIAM (1976).

[23] P. Magal and S. Ruan, Theory and Applications of Abstract semilinear Cauchy problems. Appl. Math. Sci. (2018).

[24] P. Magal, McCluskey and G.F. Webb, Lyapunov functional and global asymptotic stability for infection age model. Appl. Anal 89 (2010) 1109-1140.

[25] B. Miller, Preventive therapy for tuberculosis. Med. Clin. N. Am. 77 (1993) 1263-1275.

[26] K. Mischaikow, H. Smith and H. Thieme, Asymptotically autonomous semiflows: chain recurrence and Lyapunov functions. Trans. Am. Math. Soc. 347 (1995) 1669-1685.

[27] A. Perasso, Global stability and uniform persistence for an infection Load structured SI model with Exponential growth velocity. Commun. Pure Appl. Anal. 18 (2019) 15-32.

[28] H.l. Smith and H.R. Thieme, Dynamical Systems and population persistence. In Vol. 118 of Graduate Studies in Mathematics (2011).

[29] H.R. Thieme and C. Castillo-Chavez, How may infection age dependent infectivity affects the dynamics of HIV/AIDS. Siam J. Appl. Math. 53 (1993) 1447-1479.

[30] G.F. Webb, Theory of Nonlinear age Dependent Population Dynamics. Marcel Dekker, New York (1985).

[31] R. Xu, X. Tian and F. Zhang, Global dynamics of tuberculosis transmission model with age of infection and incomplete treatment. Adv. Differ. Equ. 2017 (2017) 242.

[32] Y. Yang, S. Ruan and D. Xia, Global stability of an age structured virus Dynamics Model with Beddington-Deangelis infection function. Math. Biosci. Eng. 12 (2015) 859-877.

[33] Z. Yui, Y. Yougguang and Z. Lu, Stability analysis of an age structured SEIRS model with time delay (2020).

\section{Subscribe to Open (S20)}

\section{A fair and sustainable open access model}

This journal is currently published in open access under a Subscribe-to-Open model (S2O). S2O is a transformative model that aims to move subscription journals to open access. Open access is the free, immediate, online availability of research articles combined with the rights to use these articles fully in the digital environment. We are thankful to our subscribers and sponsors for making it possible to publish this journal in open access, free of charge for authors.

\section{Please help to maintain this journal in open access!}

Check that your library subscribes to the journal, or make a personal donation to the S2O programme, by contacting subscribers@edpsciences.org

More information, including a list of sponsors and a financial transparency report, available at: https://www.edpsciences.org/en/maths-s2o-programme 